\title{
TRIGONOMETRISCHE REIHEN UND POTENZREIHEN MIT MEHRFACH MONOTONER KOEFFIZIENTENFOLGE*
}

\author{
BY \\ LEOPOLD FEJER \\ EINLEITUNG
}

1. In der vorliegenden Arbeit beschäftige ich mich mit Summen von der Form

$$
\sum c_{m} \sin m \theta, \quad \sum c_{m} \cos m \theta, \quad \sum c_{m} z^{m},
$$

d.h. mit trigonometrischen Reihen und Potenzreihen. Hier durchläuft $m$ entweder die Gesamtheit der nichtnegativen ganzen Zahlen $0,1,2,3, \cdots$, oder aber diese von einer bestimmten positiven ganzen Zahl $n+1$ angefangen, d.h. $m=n+1, n+2, n+3, \cdots$. Im letzteren Falle nenne ich diese Reihen Restreihen, genauer trigonometrische Restreihen oder Potenzrestreihen. Eingehend werden auch die Fälle untersucht, in denen $m$ entweder nur alle nichtnegativen geraden Zahlen, oder alle positiven ungeraden Zahlen durchläuft, bzw. diese nur von einer bestimmten angefangen. Schliesslich wird, wenn auch nur kurz, von Polynomen entsprechender Form die Rede sein, wo also die Koeffizienten $c_{m}$ von einem gewissen Index an gleich Null sind.

2. Die Grundvoraussetzung wird in der Regel die sein, dass die Koeffizientenfolge, welche die zugelassenen Indizes aufweist, und die ich immer mit

$$
c_{0}, c_{1}, c_{2}, \cdots, c_{\nu}, \cdots
$$

bezeichnen kann, eine nichtnegative monotone Nullfolge sei, $\uparrow$ und zwar entweder eine einfach monotone oder eine mehrfach monotone Nullfolge. Einfach monoton heisst eine aus nichtnegativen Gliedern bestehende Folge (2) dann, wenn auch ihre ersten Differenzen

$$
c_{0}-c_{1}, c_{1}-c_{2}, \cdots, c_{\nu}-c_{\nu+1}, \cdots
$$

eine aus durchwegs nichtnegativen Gliedern bestehende unendliche Folge bilden; zweifach monoton, wenn ausserdem noch die unendliche Folge ihrer zweiten Differenzen

$$
c_{0}-2 c_{1}+c_{2}, c_{1}-2 c_{2}+c_{3}, \cdots, c_{\nu}-2 c_{\nu+1}+c_{\nu+2}, \cdots
$$

\footnotetext{
* Presented to the Society, September 13, 1935; received by the editors February 1, 1935.

$\dagger$ Der Fall, in welchem alle $c_{\nu}=0$ sind, sei indessen ein für allemal ausgeschlossen.
} 
nichtnegativ ausfällt, u.s.w. Vollmonoton (E. Jacobsthal), oder auch total monoton (I. Schur) heisst eine Folge (2), wenn alle ihre Differenzenfolgen nichtnegativ ausfallen (d.h. $\Delta^{k} c_{\nu} \geqq 0$ für $\nu=0,1,2,3, \cdots ; k=0,1,2,3, \cdots$ ).

Auf dem Gebiete der mehrfach oder total monotonen Zahlenfolgen sind in der letzteren Zeit (1920-25) wichtige Begriffsbildungen und Resultate entstanden, die hauptsächlich von E. Jacobsthal [16], K. Knopp [18, 19, 20, 21], Th. Kaluza [17] und F. Hausdorif [11, 12] herrühren. Die höchst elementaren hierher gehörigen Sätze, die in der vorliegender Arbeit gebraucht werden, sind, mit einigen Bemerkungen versehen, im \$I zusammengestellt. Die Behandlung der Reihen (1) mit vollmonotonen Koeffizienten (2) möchte ich mir jedoch für eine spätere Veröffentlichung vorbehalten. Bei vollmonotonen $c_{\nu}$ kommt natürlich der wichtige Satz von Hausdorff zur Anwendung, nach dem in diesem Falle die Koeffizienten $c_{\nu}$ gewisse Stieltjessche Momente endlichen Intervalles sind.

3. Meine Beweismethode ist hauptsächlich auf die Positivität der Partialsummen oder der iterierten Partialsummen (Partialsummen höherer Ordnung) gewisser spezieller trigonometrischer Reihen gegründet. Man findet dies in §II dargelegt. Ich bemerke, dass diese Positivitätsbeweise sich in der Regel wieder einzig und allein auf die Nichtnegativität der Partialsummen erster Ordnung der Reihe

$$
1+2 \cos \theta+2 \cos 2 \theta+2 \cos 3 \theta+\cdots+2 \cos n \theta+\cdots
$$

für jedes reelle $\theta$, oder, was dasselbe ist, auf die Nichtnegativität der endlichen Summe

$$
\sin \theta+\sin 3 \theta+\sin 5 \theta+\cdots+\sin (2 n+1) \theta
$$

im Intervalle $0<\theta<\pi$, und für $n=0,1,2,3, \cdots$, stützen. Nichtnegativität der Summe (6) und die Abelsche Umformung einer Summe von der Form $a_{1} b_{1}+a_{2} b_{2}+\cdots+a_{n} b_{n}$ sind also die beiden Hauptstützpunkte meiner Beweisführungen.

In den Paragraphen IV-VII über die Nullstellen der Summen $\sum c_{m} \sin m \theta$, $\sum c_{m} \cos m \theta$ spielt aber noch ein drittes Beweiselement eine sehr wesentliche Rolle: Es ist dies eine originelle Schlussweise, mit deren Hilfe G. Szegö neuerdings die Nullstellen gewisser trigonometrischen Polynome in Grenzen eingeschlossen hat.* Über diese möchte ich hier einige Worte sagen.

* Herr Szegö hat mir seinen Satz über trigonometrische Polynome mit Beweis in einem Briefe vom 21. April 1934 freundlichst mitgeteilt. Vgl. seine hier unmittelbar vorangehende Arbeit [26]: Inequalities for the zeros of Legendre polynomials and related functions. Erwähnt sei noch, dass ich einiges über den Inhalt der vorliegenden Arbeit in drei Vorlesungen an einem Fortbildungskurs für Oberlehrer der Mathematik an der Budapester Universität im Juni und Juli 1934 vorgetragen habe. 
4. Die Szegösche Schlussweise stützt sich ebenfalls auf die obige Ungleichung

$$
\sum_{\nu=0}^{n} \sin (2 \nu+1) \theta \geqq 0, \quad 0<\theta<\pi,
$$

oder, was dasselbe ist, auf

$$
\sum_{\nu=0}^{n} \sin \left(\nu+\frac{1}{2}\right) \theta \geqq 0, \quad 0<\theta<2 \pi .
$$

Bevor ich nun zur eigentlichen Szegöschen Schlussweise übergehe, möchte ich, einleitend, aus der Ungleichung (8) den allereinfachsten Schluss verwandten Charakters ziehen. Es seien $c_{0}, c_{1}, c_{2}, \cdots, c_{n}$ positive Zahlen, die monoton fallen, d.h. $c_{0}>c_{1}>c_{2}>\cdots>c_{n}>0$. Durch Anwendung der gewöhnlichen Abelschen Umformung folgert man dann aus (8) unmittelbar, dass

$$
\sum_{\nu=0}^{n} c_{\nu} \sin \left(\nu+\frac{1}{2}\right) \theta>0, \quad 0<\theta<2 \pi,
$$

oder

$$
\left(\sum_{\nu=0}^{n} c_{\nu} \cos \nu \theta\right) \sin \frac{\theta}{2}+\left(\sum_{\nu=0}^{n} c_{\nu} \sin \nu \theta\right) \cos \frac{\theta}{2}>0, \quad 0<\theta<2 \pi .
$$

Aus der Ungleichung (10) folgt aber augenscheinlich, dass die Polynome $\sum_{\nu=0}^{n} c_{\nu} \cos \nu \theta$ und $\sum_{\nu=0}^{n} c_{\nu} \sin \nu \theta$ für $0<\theta<2 \pi$ nicht gleichzeitig verschwinden können. Da dies auch für $\theta=0$ der Fall ist, so ist also erwiesen, dass die beiden trigonometrischen Polynome für kein reelles $\theta$ gleichzeitig verschwinden können. Da endlich mit $c_{0}, c_{1}, \cdots, c_{n}$ auch $c_{0}, c_{1} r, \cdots, c_{n} r^{n}, 0 \leqq r<1$, monoton fallend ist, so haben wir das Resultat: Das Polynom

$$
c_{0}+c_{1} z+\cdots+c_{n} z^{n}
$$

der komplexen Veränderlichen $z$ hat keine Nullstelle für $|z| \leqq 1$, wenn $c_{0}>c_{1}>c_{2}>\cdots>c_{n}>0$. Dies ist der bekannte Eneström-Kakeyasche Satz. Der übliche Beweis dieses Satzes [durch Multiplikation mit $(1-z)$ ] ist natürlich auch höchst einfach; ich wollte hier nur zeigen, dass er auch eine unmittelbare Folge der Ungleichung (7) ist, d.h. eine Folge der Tatsache, dass die Summe der Sinusse der ersten $n$ ungeraden Multipla von $\theta$ im Intervalle $0<\theta<\pi$ stets nichtnegativ bleibt, so wie der Sinus des ersten Multiplums, d.h. $\sin \theta$ selber.

Szegö benutzt diese Tatsache nicht zum Beweis der Nichtexistenz einer gemeinsamen Nullstelle von gewissen Paaren von konjugierten trigono- 
metrischen Polynomen, sondern im Gegenteil: Er beweist die Existenz reeller Nullstellen gewisser trigonometrischen Polynome

$$
T(\theta)=\sum_{\nu=0}^{n} c_{\nu} \cos \nu \theta
$$

und schliesst diese in Grenzen ein, indem er in der folgenden Weise vorgeht. Man führe in (12) $n-\nu$ statt $\nu$ als Summationsindex ein. Dann folgt

$$
\begin{aligned}
T(\theta)= & \sum_{\nu=0}^{n} c_{n-\nu} \cos (n-\nu) \theta=\sum_{\nu=0}^{n} c_{n-\nu} \cos \left(\left(n+\frac{1}{2}\right)-\left(\nu+\frac{1}{2}\right)\right) \theta \\
= & \left(\sum_{\nu=0}^{n} c_{n-\nu} \cos \left(\nu+\frac{1}{2}\right) \theta\right) \cos \left(n+\frac{1}{2}\right) \theta \\
& +\left(\sum_{\nu=0}^{n} c_{n-\nu} \sin \left(\nu+\frac{1}{2}\right) \theta\right) \sin \left(n+\frac{1}{2}\right) \theta
\end{aligned}
$$

so dass also, wenn die Wurzeln der Gleichung $\cos \left(n+\frac{1}{2}\right) \theta=0$ mit

$$
\theta_{k}=\left(k+\frac{1}{2}\right) \frac{\pi}{n+\frac{1}{2}} \quad(k=0,1,2,3, \cdots, 2 n)
$$

bezeichnet werden, die Gleichung (13) mit einem Schlage

$$
T\left(\theta_{k}\right)=B\left(\theta_{k}\right)(-1)^{k} \quad(k=0,1,2, \cdots, 2 n)
$$

liefert. Hier bezeichnet

$$
B(\theta)=\sum_{\nu=0}^{n} c_{n-\nu} \sin \left(\nu+\frac{1}{2}\right) \theta .
$$

Ist nun $0<c_{0}<c_{1}<\cdots<c_{n}$, so ist, wie wir soeben bemerkt haben, $B(\theta)>0$ für $0<\theta<2 \pi$, so dass also aus (15)

$$
\operatorname{sgn} T\left(\theta_{k}\right)=(-1)^{k} \quad(k=0,1,2, \cdots, 2 n)
$$

folgt. Hieraus ergibt sich nach Szegö unmittelbar der folgende merkw ürdige Satz: Ist im Kosinuspolynome $c_{0}+c_{1} \cos \theta+\cdots+c_{n} \cos n \theta$

$$
0<c_{0}<c_{1}<\cdots<c_{n},
$$

so hat dieses, im Intervalle $0<\theta<2 \pi, 2 n$ voneinander verschiedene Nullstellen $t_{1}, t_{2}, t_{3}, \cdots, t_{2 n}$, und es gilt

$$
\left(k-\frac{1}{2}\right) \frac{\pi}{n+\frac{1}{2}}<t_{k}<\left(k+\frac{1}{2}\right) \frac{\pi}{n+\frac{1}{2}} \quad(k=1,2,3, \cdots, 2 n) .
$$

Ein ähnlicher Satz besteht für $c_{1} \sin \theta+c_{2} \sin 2 \theta+\cdots+c_{n} \sin n \theta$. 
Dass ein Kosinuspolynom (oder Sinuspolynom) mit positiven, monoton wachsenden Koeffizienten lauter voneinander verschiedene reelle Nullstellen besitzt, hat schon G. Pólya [22] gefunden, und aus dem Eneström-Kakeyaschen Satze abgeleitet. Wir sehen, dass Szegö durch Benutzung von $\sin \theta$ $+\sin 3 \theta+\cdots+\sin (2 n+1) \theta \geqq 0$, bis zu einer von den Koeffizienten des Kosinuspolynoms unabhängigen Abgrenzung (19) aller Nullstellen vordringen konnte.*

Die Szegösche Schlussweise in der dargelegten Form ist es nun, die ich in der vorliegenden Arbeit immer benutze, wenn ich die Nullstellen von trigonometrischen Restreihen untersuche.

5. Ich möchte noch in dieser Einleitung einige Resultate der vorliegenden Arbeit hervorheben.

Man betrachte die Reihen

$$
\begin{aligned}
& f(\theta)=c_{0}+c_{1} \cos \theta+c_{2} \cos 2 \theta+c_{3} \cos 3 \theta+\cdots+c_{n} \cos n \theta+\cdots, \\
& g(\theta)=c_{1} \sin \theta+c_{2} \sin 2 \theta+c_{3} \sin 3 \theta+\cdots \\
& \phi(\theta)=c_{1} \cos \theta+c_{2} \cos 3 \theta+c_{3} \cos 5 \theta+\cdots, \\
& \psi(\theta)=c_{1} \sin \theta+c_{2} \sin 3 \theta+c_{3} \sin 5 \theta+\cdots,
\end{aligned}
$$

* Pólya leitet aus dem Eneström-Kakeyaschen Satze mit Hilfe des Argumentsatzes der Funktionentheorie den allgemeineren Satz ab:

$$
c_{0}+c_{1} \cos \theta+\cdots+c_{n} \cos n \theta=0
$$

hat $2 n$ voneinander mod $2 \pi$ verschiedene reelle Wurzeln, falls die Wurzeln der algebraischen Gleichung

$$
c_{0}+c_{1} z+\cdots+c_{n} z^{n}=0
$$

im Innern des Einheitskreises $|z|<1$ liegen (auch für das Sinuspolynom gültig). Über die Begrenzung (Verteilung) der ausnahmslos reellen Wurzeln des Koṣinuspolynoms auf dem Einheitskreise sagt aber Pólya nichts aus. Durch geringe Modifikation der ursprünglichen Pólyaschen Beweisführung kann man indessen, wie ich bemerkt habe, auch in diesem allgemeineren Falle über die Verteilung der Wurzeln, im Szegöschen Sinne, wenigstens so viel feststellen, wie es im folgenden Satze enthalten ist:

Zerlegt man die Peripherie des Einheitskreises in $n$ gleiche Teile, so liegt an jedem Teilbogen mindestens eine Nullstelle des Kosinuspolynoms $c_{0}+c_{1} \cos \theta+\cdots+c_{n} \cos n \theta$, falls die Wurzeln der algebraischen Gleichung $c_{0}+c_{1} z+\cdots+c_{n} z^{n}$ alle im Innern des Einheitskreises liegen. (Dasselbe ist für das Sinuspolynom $c_{1} \sin \theta+c_{2} \sin 2 \theta+\cdots+c_{n} \sin n \theta$ gültig.) Der Grenzfall

$$
(1+z)_{z=e^{n} i \theta}^{n}=2^{n} \cos ^{n} \frac{\theta}{2} \cos \frac{n \theta}{2}+i 2^{n} \cos ^{n} \frac{\theta}{2} \sin \frac{n \theta}{2}
$$

zeigt, dass dieser Satz sich insofern nicht verbessern lässt, als auf einem Bogen des Einheitskreises, der kürzer als $2 \pi / n$ ist, keine Nullstelle des Kosinus- oder Sinuspolynoms zu liegen braucht. Weiter ist gültig: Auf jedem Bogen von der Länge $\pi / n$ des Einheitskreises hat entweder das Kosinuspolynom, oder das konjugierte Sinuspolynom eine Nullstelle. Bei dem Beweise dieser Sätze spielt die einfache geometrische Tatsache eine Rolle, dass ein Bogen des Einheitskreises von der Länge $l(l \leqq \pi)$ von keiner inneren Stelle der Einheitskreisfläche unter einem kleineren Winkel als $l / 2$ sichtbar ist. Übrigens gedenke ich noch in einer anderen Veröffentlichung auf die Sätze von Pólya und Szegö über trigonometrische Polynome zurückzukehren. 
(24) $\quad F(z)=c_{0}+c_{1} z+c_{2} z^{2}+\cdots$,

(25) $G(z)=c_{1} z+c_{2} z^{3}+c_{3} z^{5}+\cdots$,

wo die Koeffizientenfolgen immer nichtnegative, aber niemals identisch verschwindende Nullfolgen sind.

Ist die nichtnegative Nullfolge $c_{0}, c_{1}, c_{2}, \cdots$ zweifach monoton (es genügt $2 c_{0}$ statt $c_{0}$ zu nehmen), so ist $f(\theta)$ für jedes $\theta$ nichtnegativ (\$III). Ist $c_{1}, c_{2}$, $c_{3}, \cdots$ vierfach monoton, so ist $f(\theta)$ im Intervalle $0<\theta<\pi$ monoton fallend. Ist $c_{1}, c_{2}, c_{3}, \cdots$ nur dreifach monoton, so braucht $f(\theta)$ im Intervalle $(0, \pi)$ nicht monoton fallend zu sein (§III). Ist $c_{1}, c_{2}, c_{3}, \cdots$ zweifach monoton, so ist $g(\theta)$ für $0<\theta<\pi$ positiv ( $(I I I)$. Ist $c_{1}, c_{2}, c_{3}, \cdots$ vierfach monoton, so ist $g(\theta)$ im Intervalle $\pi / 2<\theta<\pi$ monoton fallend. Ist $c_{1}, c_{2}, c_{3}, \cdots$ dreifach monoton, so ist $\phi(\theta)$ in $(0, \pi / 2)$ positiv, in $(\pi / 2, \pi)$ negativ, u.s.w., es hat also das Vorzeichen von $\cos \theta$; d.h. sgn $\phi(\theta)=\operatorname{sgn} \cos \theta$ (§III). Weiter ist $\phi(\theta)$ auch monoton fallend in $(0, \pi)$. Ist $c_{1}, c_{2}, c_{3}, \cdots$ einfach monoton, so ist $\operatorname{sgn} \psi(\theta)=\operatorname{sgn} \sin \theta(\S \mathrm{III})$. Ist $c_{0}, c_{1}, c_{2}, \cdots$ dreifach monoton, so ist für den Rest der Potenzreihe von $F(z)$ die Ungleichung $\left|c_{n+1} z^{n+1}+c_{n+2} z^{n+2}+\cdots\right|$ $\leqq|F(z)|$ gültig für $|z| \leqq 1, z \neq 1, n=0,1,2,3, \cdots$, woraus $\left|s_{n}(z)\right|=\mid c_{0}$ $+c_{1} z+\cdots+c_{n} z^{n}|\leqq 2| F(z) \mid$ folgt, $|z| \leqq 1, z \neq 1, n=0,1,2,3, \cdots$ (§VIII). Ist $c_{1}, c_{2}, c_{3}, \cdots$ vierfach monoton, so ist die Funktion $F(z)$ schlicht für $|z|<1$ (§IX). Ist $c_{1}, c_{2}, c_{3}, \cdots$ vierfach monoton, so ist jeder Rest von $G(z)$ absolut $\leqq|G(z)|$ für $|z| \leqq 1, z \neq 1$; ist $c_{1}, c_{2}, c_{3}, \cdots$ dreifach monoton, so ist $G(z)$ schlicht für $|z|<1$.

Von meinen Sätzen über die Abgrenzung der Nullstellen von Restreihen sei hier nur der folgende angeführt:

Ist die Koeffizientenfolge $c_{0}, c_{1}, c_{2}, \cdots, c_{\nu}, \cdots$ der trigonometrischen Reihe

$$
h(\theta)=\sum_{\nu=0}^{\infty} c_{\nu} \sin (n+2 \nu+1) \theta=c_{0} \sin (n+1) \theta+c_{1} \sin (n+3) \theta+\cdots
$$

eine dreifach monotone Nullfolge, so hat die für $0<\theta<\pi$ stetige Funktion $h(\theta)$ im Intervalle $0<\theta<\pi$ mindestens $n$ Nullstellen $t_{1}, t_{2}, \cdots, t_{n}$. Für die im Intervalle $0<\theta \leqq \pi / 2$ gelegenen Nullstellen gelten die Grenzen

$$
\left(k-\frac{1}{2}\right) \frac{\pi}{n}<t_{k}<k \frac{\pi}{n+1} \quad\left(k=1,2,3, \cdots, n^{\prime}=\left[\frac{n+1}{2}\right]\right) .
$$

[Ist $n$ ungerade, so muss statt der letzten dieser Ungleichungen

$$
\left(n^{\prime}-\frac{1}{2}\right) \frac{\pi}{n}=t_{n^{\prime}}=n^{\prime} \frac{\pi}{n+1}\left(=\frac{\pi}{2}\right)
$$

geschrieben werden.] Für die übrigen Nullstellen $t_{k}$, die doch $z u$ den obigen 
i.B. auf $\pi / 2$ symmetrisch liegen, gelten die $z$ u den obigen i.B. auf $\pi / 2$ symmetrischen Grenzen ( $\$ I V)$.

A. Hurwitz bemerkt in seiner Arbeit [15] dass aus der blossen Tatsache, dass die unendliche Sinusreihe des $n$ ten Legendreschen Polynoms $P_{n}(\cos \theta)$ [die Heinesche Sinusreihe von $P_{n}(\cos \theta)$ ] die Form

$$
P_{n}(\cos \theta)=\sum_{\nu=0}^{\infty} c_{\nu} \sin (n+2 \nu+1) \theta=c_{0} \sin (n+1) \theta+c_{1} \sin (n+3) \theta+\cdots
$$

hat, d.h. dass in ihr die Koeffizienten von $\sin \theta, \sin 2 \theta, \cdots, \sin n \theta$ gleich Null sind, ohne weiteres die Existenz von $n$ verschiedenen Nullstellen von $P_{n}(\cos \theta)$ im Intervalle $(0, \pi)$ folgt. Er gewinnt dies aus der Verallgemeinerung eines Sturmschen Satzes bezüglich trigonometrischer Polynome, die mit $c_{0} \sin (n+1) \theta+\cdots$ anfangen, auf Reihen der gleichen Form. Ich fand nun, dass die Koeffizienten $c_{0}, c_{1}, c_{2}, \cdots$ in der Heineschen Sinusreihe eine dreifach monotone (ja sogar eine vollmonotone) Folge bilden. Auf Grund meines obigen Satzes, und durch blosse Ansicht der Koeffizientenfolge der Heineschen Sinusreihe folgen somit für die Nullstellen von $P_{n}(\cos \theta)$ die Schranken (27), d.h. die sog. Markoff-Stieltjesschen Nullstellenschranken.

6. Es mag vielleicht Interesse finden, wenn ich in dieser Einleitung noch zeige, wie kurz und natürlich bewiesen werden kann, dass die Koeffizientenfolge der Heineschen Sinusreihe von $P_{n}(\cos \theta)$ (eine sehr bemerkenswerte Entwicklung), vollmonoton ist.

Ist

$$
(1-z)^{-1 / 2}=\sum_{\nu=0}^{\infty} \alpha_{\nu} z^{\nu}=\sum_{\nu=0}^{\infty} \frac{1 \cdot 3 \cdot 5 \cdots(2 \nu-1)}{2 \cdot 4 \cdot 6 \cdots 2 \nu} z^{\nu},
$$

so wird bekanntlich

$$
P_{n}(\cos \theta)=\sum_{k=0}^{n} \alpha_{k} e^{k i \theta} \alpha_{n-k} e^{-(n-k) i \theta}=\sum_{k=i}^{n} \alpha_{k} \alpha_{n-k e^{(2 i-n) i \theta}},
$$

woraus

$$
P_{n}(\cos \theta)=\sum_{k=0}^{n} \alpha_{k} \alpha_{n-k} \cos (n-2 k) \theta,
$$

so dass $P_{n}(\cos \theta)$ ein Kosinuspolynom $n$ ter Ordnung ist. Suchen wir nun die Sinusreihe von $P_{n}(\cos \theta)$ :

$$
b_{1} \sin \theta+b_{2} \sin 2 \theta+\cdots+b_{n} \sin n \theta+b_{n+1} \sin (n+1) \theta+\cdots .
$$

Ich behaupte zunächst, dass 


$$
b_{1}=b_{2}=\cdots=b_{n}=0
$$

ist. Tatsächlich gilt nach Fourier

$$
\begin{aligned}
b_{\nu} & =\frac{2}{\pi} \int_{0}^{\pi} P_{n}(\cos \theta) \sin \nu \theta d \theta=\frac{2}{\pi} \int_{0}^{\pi} P_{n}(\cos \theta) \cdot \frac{\sin \nu \theta}{\sin \theta} \sin \theta d \theta \\
& =\frac{2}{\pi} \int_{-1}^{+1} P_{n}(x) U_{\nu-1}(x) d x,
\end{aligned}
$$

wo $U_{\nu-1}(x)$ ein Polynom in $x$ von $(\nu-1)$ tem Grade bezeichnet, nämlich $\sin \nu \theta / \sin \theta$ als Funktion von $\cos \theta=x$. Wegen der Orthogonalitätseigenschaft von $P_{n}(x)$ gilt also (34) tatsächlich. Da weiter $P_{n}(\cos (\pi-\theta))=P_{n}(\cos \theta)$ bzw. $=-P_{n}(\cos \theta)$ ist, je nachdem $n$ gerade oder ungerade ist, so haben wir für die Sinusreihe von $P_{n}(\cos \theta)$ in beiden Fällen die Form

$$
\begin{aligned}
P_{n}(\cos \theta) & =\sum_{\nu=0}^{\infty} c_{\nu}^{*} \sin (n+2 \nu+1) \theta \\
& =c_{0}^{*} \sin (n+1) \theta+c_{1}^{*} \sin (n+3) \theta+\cdots
\end{aligned}
$$

erhalten.

Um hier die Koeffizienten $c_{\nu}^{*}$ zu bestimmen, habe ich, mit Rücksicht auf (32), nur $\cos (n-2 k) \theta$ in eine Sinusreihe zu entwickeln. Da diese Funktion dieselbe Symmetrieeigenschaft wie $P_{n}(\cos \theta)$ hat, so besitzt ihre Sinusreihe die Form

$$
\begin{aligned}
\cos (n-2 k) \theta & =\cdots+\sum_{\nu=0}^{\infty} \beta_{\nu} \sin (n+2 \nu+1) \theta \\
& =\cdots+\beta_{0} \sin (n+1) \theta+\beta_{1} \sin (n+3) \theta+\cdots ;
\end{aligned}
$$

die Koeffizienten von $\sin \theta, \sin 2 \theta, \cdots, \sin n \theta$ haben hier kein Interesse für uns, da sie, wie schon erwiesen, nach der in (32) geforderten Addition ohnehin wegfallen. Nun ist, mit Rücksicht auf (37), nach Fourier

$$
\begin{aligned}
\beta_{\nu} & =\frac{2}{\pi} \int_{0}^{\pi} \cos ((n-2 k) \theta) \sin ((n+2 \nu+1) \theta) d \theta \\
& =\frac{2}{\pi}\left(\frac{1}{2(n-k)+2 \nu+1}+\frac{1}{2 k+2 \nu+1}\right) \quad(\nu=0,1,2, \cdots) .
\end{aligned}
$$

Also ist, mit Rücksicht auf (38), (37), (36) und (32),

$$
c_{\nu}^{*}=\frac{2}{\pi} \sum_{k=0}^{n} \alpha_{k} \alpha_{n-k}\left(\frac{1}{2(n-k)+2 \nu+1}+\frac{1}{2 k+2 \nu+1}\right) .
$$

Man hat aber 


$$
\sum_{k=0}^{n} \alpha_{k} \alpha_{n-k} \frac{1}{2(n-k)+2 \nu+1}=\sum_{k=0}^{n} \alpha_{k} \alpha_{n-k} \frac{1}{2 k+2 \nu+1},
$$

da die eine dieser Summen aus der anderen durch Ersetzen von $k$ durch $n-k$ hervorgeht. Also wird schliesslich $\dagger$

$$
\begin{aligned}
c_{\nu}^{*} & =\frac{4}{\pi} \sum_{k=0}^{n} \alpha_{k} \alpha_{n-k} \frac{1}{2 k+2 \nu+1} \\
& =\frac{4}{\pi}\left\{\frac{\alpha_{0} \alpha_{n}}{2 \nu+1}+\frac{\alpha_{1} \alpha_{n-1}}{2 \nu+3}+\frac{\alpha_{2} \alpha_{n-2}}{2 \nu+5}+\cdots+\frac{\alpha_{n} \alpha_{0}}{2 \nu+2 n+1}\right\},
\end{aligned}
$$

wo $\alpha_{k}$, ich wiederhole es, den Wert

$$
\alpha_{k}=\frac{1 \cdot 3 \cdots(2 k-1)}{2 \cdot 4 \cdots 2 k}
$$

bezeichnet.

Aus dieser Form (41) der Heineschen Sinuskoeffizienten $c_{\nu}^{*}$ ist aber evident, dass $c_{0}^{*}, c_{1}^{*}, \ldots$ eine positive vollmonotone Nullfolge ist, da doch $1 /(2 k+2 \nu+1), \nu=0,1,2, \cdots$, für jeden einzelnen der $(n+1)$ Werte $k=0$, $1, \cdots, n$, eine solche ist und $\alpha_{0}, \alpha_{1}, \cdots, \alpha_{n}$ positive Zahlen bezeichnen.

\section{I. ÜBer Summen UND Differenzen hÖHERER ORDNuNG}

1. Ist

$$
u_{0}+u_{1}+u_{2}+\cdots+u_{n}+\cdots
$$

eine beliebige unendliche Reihe, so heissen die Folgen

(2) $s_{n}{ }^{(0)}=\sum_{\nu=0}^{n} u_{\nu}, s_{n}{ }^{(1)}=\sum_{\nu=0}^{n} s_{\nu}{ }^{(0)}, s_{n}{ }^{(2)}=\sum_{\nu=0}^{n} s_{\nu}{ }^{(1)}, \cdots \quad(n=0,1,2,3, \cdots)$

die Partialsummen 0ter, 1ter, 2ter, . . Ordnung der unendlichen Reihe (1). Sind die Glieder der Folge $s_{n}^{(k)}$ der Partialsummen $k$ ter Ordnung einer unendlichen Reihe (1) nichtnegativ, so sage ich, dass die Reihe von der kten Ordnung nichtnegativ (kurz, $k$ ter Ordnung positiv) ist. Ist eine Reihe positiv von der $k$ ten Ordnung, so ist sie natürlich auch positiv von der $k^{\prime}$ ten Ordnung, falls $k^{\prime}>k$. Die kleinste Ordnung $k$, nach welcher (1) positiv ist, heisse ihre wahre Ordnung. Diese kann für eine Reihe auch $+\infty$ sein.

$\dagger$ Aus dieser merkwürdigen Form der $c_{\nu}^{*}$ ergibt sich u.a. ohne weiteres die Form (6), (7) in §VI, da z.B. eine Partialbruchzerlegung die Gleichung

$$
c_{\nu}=(\pi / 4) c_{\nu}^{*}=\sum_{k=0}^{n} \frac{\alpha_{k} \alpha_{n-k}}{2 k+2 \nu+1}=\frac{(2 \nu+2)(2 \nu+4) \cdots(2 \nu+2 n)}{(2 \nu+1)(2 \nu+3) \cdots(2 \nu+2 n-1)(2 \nu+2 n+1)}
$$

liefert. 
Ist der Konvergenzradius der Potenzreihe

$$
F(r)=\sum_{\nu=0}^{\infty} u_{\nu} r^{\nu}
$$

positiv, so heisst die Funktion $F(r)$ die erzeugende Funktion der Gliederfolge $u_{0}, u_{1}, u_{2}, \ldots$; dann stellt

$$
(1-r)^{-k-1} F(r)=\sum_{\nu=0}^{\infty} s_{\nu}{ }^{(k)} r^{\nu}
$$

die erzeugende Funktion der Folge der $k$ ten Partialsummen der Reihe (1) dar. Es ist also

$$
\begin{aligned}
& s_{n}{ }^{(k)}=\left(\begin{array}{c}
n+k \\
k
\end{array}\right) u_{0}+\left(\begin{array}{c}
n+k-1 \\
k
\end{array}\right) u_{1}+\left(\begin{array}{c}
n+k-2 \\
k
\end{array}\right) u_{2} \\
&+\cdots+\left(\begin{array}{l}
k \\
k
\end{array}\right) u_{n}(n=0,1,2,3, \cdots ; k=0,1,2,3, \cdots) .
\end{aligned}
$$

2. Es sei

$$
v_{0}, v_{1}, v_{2}, \cdots, v_{n}, \cdots
$$

eine beliebige unendliche Folge. Ferner sei

(7)

$$
\begin{aligned}
& \Delta^{0} v_{n}=v_{n}, \\
& \Delta^{1} v_{n}=\Delta v_{n}=v_{n}-v_{n+1}, \\
& \Delta^{2} v_{n}=\Delta\left(\Delta^{1} v_{n}\right)=v_{n}-2 v_{n+1}+v_{n+2}, \\
& \Delta^{k} v_{n}=\Delta\left(\Delta^{k-1} v_{n}\right)=\left(\begin{array}{l}
k \\
0
\end{array}\right) v_{n}-\left(\begin{array}{l}
k \\
1
\end{array}\right) v_{n+1}+\left(\begin{array}{l}
k \\
2
\end{array}\right) v_{n+2} \\
& -\cdots+(-1)^{k}\left(\begin{array}{l}
k \\
k
\end{array}\right) v_{n+k} \quad(n=0,1,2,3, \cdots) .
\end{aligned}
$$

Hier heisst $\Delta^{k} v_{n}$ die Differenz kter Ordnung mit dem Index $n$ der Folge (6). Sind für eine Folge (6) bis zur Ordnung $k$ inklusive alle Differenzen nichtnegativ, d.h. bestehen die Folgen $\Delta^{0} v_{n}, \Delta^{1} v_{n}, \Delta^{2} v_{n}, \cdots, \Delta^{k} v_{n}, n=0,1,2,3, \cdots$, aus lauter nichtnegativen Zahlen, so heisst die Folge (6) $k$-fach monoton. Ist dies für jede Ordnung $k$ gültig, so heisst (6) vollmonoton, oder auch total monoton. Ist die Folge (6) $k$-fach monoton bzw. vollmonoton, so ist auch jede ihrer Restfolgen $v_{n+1}, v_{n+2}, v_{n+3}, \cdots k$-fach monoton bzw. vollmonoton. Sind beide Folgen $v_{n}, w_{n} k$-fach monoton, so gilt dasselbe auch für die Fol- 
gen $v_{n}+w_{n}, v_{n} w_{n}$. Das letztere folgt aus der für jede Ordnung geltenden bekannten wichtigen Formel*

$$
\Delta^{k}\left(v_{n} w_{n}\right)=\sum_{\nu=0}^{k}\left(\begin{array}{l}
k \\
\nu
\end{array}\right) \Delta^{v} v_{n} \Delta^{k-\nu} w_{n+\nu}
$$

Es ist also

$$
\begin{aligned}
& \Delta^{0}\left(v_{n} w_{n}\right)=v_{n} w_{n}, \\
& \Delta^{1}\left(v_{n} w_{n}\right)=v_{n} \Delta^{1} w_{n}+\left(\Delta^{1} v_{n}\right) w_{n+1}, \\
& \Delta^{2}\left(v_{n} w_{n}\right)=v_{n} \Delta^{2} w_{n}+2\left(\Delta^{1} v_{n}\right) \Delta^{1} w_{n+1}+\left(\Delta^{2} v_{n}\right) w_{n+2}, \\
& \Delta^{3}\left(v_{n} w_{n}\right)=v_{n} \Delta^{3} w_{n}+3\left(\Delta^{1} v_{n}\right) \Delta^{2} w_{n+1}+3\left(\Delta^{2} v_{n}\right) \Delta^{1} w_{n+2}+\left(\Delta^{3} v_{n}\right) w_{n+3},
\end{aligned}
$$

3. Ist

$$
F(r)=\sum_{\nu=0}^{\infty} v_{\nu} r^{\nu}
$$

die erzeugende Funktion der Folge $v_{0}, v_{1}, v_{2}, \cdots$, und ist

$$
(1-r)^{k} F(r)=\cdots+\lambda_{n} r^{n}+\lambda_{n+1} r^{n+1}+\cdots+\lambda_{n+k} r^{n+k}+\cdots,
$$

so hat man

$$
\Delta^{k} v_{n}=(-1)^{k} \lambda_{n+k},
$$

d.h. $(-1)^{k} \Delta^{k} v_{n}$ stimmt mit dem $(n+k)$ ten Koeffizienten in der Entwicklung von $(1-r)^{k} F(r)$ nach Potenzen von $r$ überein $(n, k=0,1,2,3, \cdots)$.

\section{Beispiel:}

$$
v_{0}=1, \quad v_{n}=\frac{\rho(\rho+1)(\rho+2) \cdots(\rho+n-1)}{1 \cdot 2 \cdot 3 \cdots n} \quad(n=1,2,3, \cdots) .
$$

Hier ist $F(r)=(1-r)^{-\rho}$, also $(1-r)^{k} F(r)=(1-r)^{-(p-k)}$. Folglich gilt

$$
\Delta^{k} v_{n}=(-1)^{k} \lambda_{n+k}=\frac{(1-\rho)(2-\rho) \cdots(k-\rho)}{1 \cdot 2 \cdots k} \cdot \frac{\rho(\rho+1) \cdots(\rho+n-1)}{(k+1)(k+2) \cdots(k+n)} \text {. }
$$

Ist also $0<\rho<1$, so hat man für die Folge (13): $\Delta^{k} v_{n}>0$, wo $n, k=0,1,2$, $3, \cdots$, d.h. sie ist vollmonoton.

4. Recht nützlich ist auch der folgende Satz.

Es sei

* Diese Formel ergibt $\Delta^{k}\left(v_{n} w_{n}\right) \geqq v_{n} \Delta^{k} w_{n}$ und natürlich auch $\geqq w_{n} \Delta^{k} v_{n}$, falls beide Folgen $v_{n}, w_{n}$ $k$-fach monoton sind. 


$$
\left(1-q_{1}\right)\left(1-q_{2}\right) \cdots\left(1-q_{n}\right) \cdots
$$

ein unendliches Produkt, in welchem die $q_{1}, q_{2}, \cdots, q_{n}, \cdots$ nichtnegative Zahlen bezeichnen, die 1 nicht überschreiten. Ist dann die Folge $q_{1}, q_{2}, \cdots$, $q_{n}, \cdots k$-fach monoton, so ist die unendliche Folge der Teilprodukte

$$
Q_{1}, Q_{2}, \cdots, Q_{n}, \cdots,
$$

wo

$$
Q_{n}=\prod_{\nu=1}^{n}\left(1-q_{v}\right)
$$

mindestens $(k+1)$-fach monoton.

Es ist gewiss $\Delta^{0} Q_{n}=Q_{n} \geqq 0$. Weiter ist

$$
\Delta^{1} Q_{n}=Q_{n}-Q_{n+1}=Q_{n}\left(1-\left(1-q_{n+1}\right)\right),
$$

d.h.

$$
\Delta^{2} Q_{n}=Q_{n} q_{n+1} .
$$

Aus dieser Gleichung (18) erhalten wir durch sukzessive Differenzbildung, mit Hilfe der Formel (8), die folgende Kette von Gleichungen:

$$
\begin{aligned}
& \Delta^{2} Q_{n}=Q_{n} \Delta^{1} q_{n+1}+\left(\Delta^{1} Q_{n}\right) q_{n+2}, \\
& \Delta^{3} Q_{n}=Q_{n} \Delta^{2} q_{n+1}+2\left(\Delta^{1} Q_{n}\right) \Delta^{1} q_{n+2}+\left(\Delta^{2} Q_{n}\right) q_{n+3},
\end{aligned}
$$

$$
\Delta^{k+1} Q_{n}=Q_{n} \Delta^{k} q_{n+1}+\left(\begin{array}{c}
k \\
1
\end{array}\right)\left(\Delta^{2} Q_{n}\right) \Delta^{k-1} q_{n+2}+\cdots+\left(\begin{array}{c}
k \\
k
\end{array}\right)\left(\Delta^{k} Q_{n}\right) q_{n+k+1} .
$$

Aus dieser Gleichungskette folgt aber der behauptete Satz unmittelbar.

II. Über die Ordnung der Positivität der Summen verschiedener ORDNUNG VON EINIGEN ELEMENTAREN TRIGONOMETRISCHEN

\section{REIHEN}

1. Setzt $\operatorname{man} z=r e^{i \theta}$ in die Reihen

$$
\frac{1+z}{1-z}=1+2 \sum_{\nu=1}^{\infty} z^{\nu}, \quad \frac{z}{1-z^{2}}=\sum_{\nu=0}^{\infty} z^{2 v+1},
$$

so folgen durch Spaltung des reellen Teiles vom imaginären vier Reihen, die zusammen mit ihren Ableitungen nach $\theta$ folgendermassen lauten:

(1) $F_{1}(r)=\frac{1-r^{2}}{1-2 r \cos \theta+r^{2}}=1+2 \sum_{\nu=1}^{\infty} r^{\nu} \cos \nu \theta$, 
(2) $F_{2}(r)=\frac{\sin \theta}{1-2 r \cos \theta+r^{2}}=\sum_{\nu=0}^{\infty} r^{\nu} \sin (\nu+1) \theta$,

(3) $F_{3}(r)=\frac{(1-r) \cos \theta}{1-2 r \cos 2 \theta+r^{2}}=\sum_{\nu=0}^{\infty} r^{\nu} \cos (2 \nu+1) \theta$,

(4) $F_{4}(r)=\frac{(1+r) \sin \theta}{1-2 r \cos 2 \theta+r^{2}}=\sum_{\nu=0}^{\infty} r^{\nu} \sin (2 \nu+1) \theta$,

(5) $F_{5}(r)=\frac{\cos \theta-2 r+(\cos \theta) r^{2}}{\left(1-2 r \cos \theta+r^{2}\right)^{2}}=\sum_{\nu=0}^{\infty} r^{\nu}(\nu+1) \cos (\nu+1) \theta$,

(6) $F_{6}(r)=\frac{\left(1-r^{2}\right) \sin \theta}{\left(1-2 r \cos \theta+r^{2}\right)^{2}}=\sum_{\nu=0}^{\infty} r^{\nu}(\nu+1) \sin (\nu+1) \theta$,

(7) $F_{7}(r)=\frac{(1+r)\left(1-2\left(3-2 \cos ^{2} \theta\right) r+r^{2}\right) \cos \theta}{\left(1-2 r \cos 2 \theta+r^{2}\right)^{2}}=\sum_{\nu=0}^{\infty} r^{\nu}(2 \nu+1) \cos (2 \nu+1) \theta$,

(8) $F_{8}(r)=\frac{(1-r)\left(1+2\left(1+2 \cos ^{2} \theta\right) r+r^{2}\right) \sin \theta}{\left(1-2 r \cos 2 \theta+r^{2}\right)^{2}}=\sum_{\nu=0}^{\infty} r^{\nu}(2 \nu+1) \sin (2 \nu+1) \theta$.

2. Die von unserem Standpunkte aus "ungünstigste" unter diesen Reihen ist $F_{7}(r)$. Für ihren Zähler

$$
f_{7}(r)=(1+r)\left(1-2\left(3-2 \cos ^{2} \theta\right) r+r^{2}\right) \cos \theta
$$

gilt nämlich $f_{7}(0)=\cos \theta, f_{7}(1)=-8 \sin ^{2} \theta \cos \theta$, so dass $F_{7}(r)$ für $0 \leqq r<1$ sowohl positive als auch negative Werte annimmt, welchen Wert auch $\theta$ habe; nur die Werte $\theta=0, \pi / 2,2 \pi / 2,3 \pi / 2$ sind Ausnahmen. Daraus folgt, dass $(1-r)^{-k} F_{7}(r)$ sowohl positive als auch negative Koeffizienten haben muss, wenn $\theta$ beliebig, nur von $0, \pi / 2, \pi, 3 \pi / 2$ verschieden ist, und zwar für jeden nichtnegativen ganzzahligen Wert von $k$. Die Reihe

(9) $\cos \theta+3 \cos 3 \theta+5 \cos 5 \theta+\cdots+(2 \nu-1) \cos (2 \nu-1) \theta+\cdots$

ist also von keiner (noch so hohen) Ordnung nichtnegativ oder nichpositiv (ausgenommen $\theta=0, \pi / 2, \pi, 3 \pi / 2$ ).

Da, $f_{5}(r)=\cos \theta-2 r+(\cos \theta) r^{2}$ gesetzt, $f_{5}(0)=\cos \theta, f_{5}(1)=-4 \sin ^{2}(\theta / 2)$, so ist die Reihe

$$
\cos \theta+2 \cos 2 \theta+3 \cos 3 \theta+\cdots+\nu \cos \nu \theta+\cdots
$$

für $-\pi / 2<\theta<\pi / 2$ weder nichtpositiv, noch nichtnegativ $k$ ter Ordnung, wie gross auch die Ordnungszahl $k$ sei (ausgenommen $\theta=0$ ). Ist aber $\pi / 2<\theta$ $<3 \pi / 2$, so ist die Reihe (10), wie unten gezeigt wird, von der dritten Ordnung negativ.

3. Was nun die übrigen sechs trigonometrischen Reihen anbelangt, so 
sind diese in geeigneten Intervallen alle nichtnegativ oder nichtpositiv von einer gewissen Ordnung. [Vgl. besonders Fejér 5, 7, 9, 10.]

Zunächst ist die Reihe

$$
1+2 \cos \theta+2 \cos 2 \theta+\cdots+2 \cos \nu \theta+\cdots
$$

für jeden reellen Wert von $\theta$ nichtnegativ von der ersten Ordnung. In der Tat ist hier

$$
s_{n}^{(1)}(\theta)=(n+1)+n \cdot 2 \cos \theta+(n-1) \cdot 2 \cos 2 \theta+\cdots+1 \cdot 2 \cos n \theta .
$$

Diese Summe $s_{n}^{(1)}(\theta)$ ist gleich $(\sin (\theta / 2))^{-2}(\sin [(n+1) \theta / 2])^{2}$, also nichtnegativ für jedes* $\theta$. Eine andere, von Toeplitz herrührende, Darstellung,

$$
\begin{aligned}
(n+ & 1)+2 n \cos \theta+2(n-1) \cos 2 \theta+\cdots+2 \cdot 1 \cdot \cos n \theta \\
& =\left(\sum_{\nu=0}^{n} \cos \nu \theta\right)^{2}+\left(\sum_{\nu=0}^{n} \sin \nu \theta\right)^{2}=\left|1+z+z^{2}+\cdots+z^{n}\right|_{z=e^{i \theta}}^{2} \\
& =\left|\frac{1-z^{n+1}}{1-z}\right|_{z=c^{i \theta}}^{2},
\end{aligned}
$$

setzt dies auch in Evidenz; wir sehen, dass $s_{n}^{(1)}(\theta)$ immer positiv ist, und nur für die Argumente der $(n+1)$ ten Einheitswurzeln verschwindet (die Wurzel $z=1$, d.h. $\theta=0$, ausgenommen). Die Reihe (11) ist somit für jeden reellen Wert von $\theta$ von der ersten Ordnung nichtnegativ.

4. Merkwürdig ist es nun, dass auf Grund der soeben bewiesenen Tatsache, nach welcher die Koeffizienten der Potenzreihe von $(1-r)^{-2} F_{1}(r)$ für jeden Wert von $\theta$ nichtnegativ sind, die Diskussion der übrigen Reihen fast ohne Rechnung erfolgen kann.

\section{Wegen}

$$
F_{2}(r)=(\sin \theta)\left(1-r^{2}\right)^{-1} F_{1}(r)
$$

und da $\left(1-r^{2}\right)^{-1}=1+r^{2}+r^{4}+\cdots$ nichtnegative Koeffizienten besitzt, folgt sofort, dass die Reihe

$$
\sin \theta+\sin 2 \theta+\cdots+\sin n \theta+\cdots
$$

für $0<\theta<\pi$ entschieden positiv, für $\pi<\theta<2 \pi$ entschieden negativ von der ersten Ordnung ist† (Satz von Lukács).

* Für $n=1$ ist $s_{1}^{(1)}(\theta)=(\sin (\theta / 2))^{-2}(\sin \theta)^{2}=4 \cos ^{2}(\theta / 2)$, also positiv in $0 \leqq \theta \leqq 2 \pi$, nur $\theta=\pi$ ausgenomen.

$\dagger$ Es sei hervorgehoben, dass die $s_{n}^{(1)}(\theta)$ der Reihe (15) für $0<\theta<\pi$ entschieden positiv sind. Da nämlich

$$
(1-r)^{-2} F_{1}(r)=1+4 \cos ^{2}(\theta / 2) r+\cdots,\left(1-r^{2}\right)^{-1}=1+r^{2}+r^{4}+\cdots,
$$

so sind im Produkte die Koeffizienten vom geraden Index $\geqq 1$, die vom ungeraden Index $\geqq 4 \cos ^{2}(\theta / 2)$. (14) liefert endlich, dass die $s_{n}{ }^{(1)}(\theta)$ der Reihe (15) für $0<\theta<\pi$ die gemeinsame positive Minorante $\sin \theta \cos ^{2}(\theta / 2)$ haben. 
Da weiter

$$
F_{3}(r)=(\cos \theta)(1-r)\left(1-r^{2}\right)^{-1} F_{1}(r, 2 \theta)
$$

so ist

$$
(1-r)^{-3} F_{3}(r)=(\cos \theta)\left(1-r^{2}\right)^{-1}(1-r)^{-2} F_{1}(r, 2 \theta) .
$$

Ich habe also erhalten, dass die Reihe

$$
\cos \theta+\cos 3 \theta+\cos 5 \theta+\cdots
$$

für $0 \leqq \theta<\pi / 2$ entschieden positiv, für $\pi / 2<\theta \leqq \pi$ entschieden negativ von der zweiten Ordnung ist.

Da

$$
F_{4}(r)=(\sin \theta)(1-r)^{-1} F_{1}(r, 2 \theta),
$$

so ist es klar, dass die Reihe

$$
\sin \theta+\sin 3 \theta+\sin 5 \theta+\cdots
$$

für $0 \leqq \theta \leqq \pi$ von der nullten Ordnung nichtnegativ ist.

$\mathrm{Da}$

$$
F_{6}(r)=(\sin \theta)\left(1-r^{2}\right)^{-1}\left(F_{1}(r)\right)^{2},
$$

so ist

$$
(1-r)^{-4} F_{6}(r)=(\sin \theta)\left(1-r^{2}\right)^{-1}\left((1-r)^{-2} F_{1}(r)\right)^{2} .
$$

Dies liefert, dass die Reihe

$$
\sin \theta+2 \sin 2 \theta+3 \sin 3 \theta+\cdots
$$

im Intervalle $0<\theta<\pi$ von der dritten Ordnung positiv ist.

Bemerkung. Da

$$
(1-r)^{-2} F_{1}(r)=1+\left(\frac{\sin (2 \theta / 2)}{\sin (\theta / 2)}\right)^{2} r+\cdots=1+4 \cos ^{2}(\theta / 2) r+\cdots,
$$

so ist

$$
\left((1-r)^{-2} F_{1}(r)\right)^{2}=1+8 \cos ^{2}(\theta / 2) r+\cdots .
$$

Durch Multiplikation dieser Reihe mit $\left(1-r^{2}\right)^{-1}=1+r^{2}+r^{4}+\cdots$ erhalte ich nun eine Potenzreihe, deren Koeffizienten aus lauter nichtnegativen Addenden zusammengesetzt sind. Ist der Index des Koeffizienten gerade, so ist die 1 , ist er ungerade, so ist $8 \cos ^{2}(\theta / 2)$ einer dieser Addenden. Daraus folgt, mit Rücksicht auf (22), dass die Summen dritter Ordnung $s_{n}^{(3)}(\theta)$ der Reihe (23) im Intervalle $0<\theta<\pi$ die gemeinsame Minorante $\sin \theta \cos ^{2}(\theta / 2)$ haben. 
$\mathrm{Da}$

$$
F_{8}(r)=(1-r)(\sin \theta)\left(1+2\left(1+2 \cos ^{2} \theta\right) r+r^{2}\right)\left(1-r^{2}\right)^{-2}\left(F_{1}(r, 2 \theta)\right)^{2},
$$

so ist

$$
\begin{aligned}
(1-r)^{-5} F_{8}(r)=\sin \theta(1+ & \left.2\left(1+2 \cos ^{2} \theta\right) r+r^{2}\right) \\
& \left(1-r^{2}\right)^{-2}\left((1-r)^{-2} F_{1}(r, 2 \theta)\right)^{2},
\end{aligned}
$$

woraus sich die Positivität vierter Ordnung der Reihe

$$
\sin \theta+3 \sin 3 \theta+5 \sin 5 \theta+\cdots
$$

für $0<\theta<\pi$ ergibt. Hier ist jedoch 4 nicht die wahre Ordnung. Da die doppelt genommene Reihe (26) entsteht, indem wir in der Reihe (23) $\theta$ durch $\pi-\theta$ ersetzen und die so entstandene Reihe zu (23) addieren, so ist es klar, dass (26) für $0<\theta<\pi$ positiv von der dritten Ordnung ist. Ich habe bewiesen [Fejér 10], dass sie im Intervalle $0<\theta<\pi$ sogar von der zweiten Ordnung positiv ist (wenn man den Mittelpunkt $\theta=\pi / 2$ bei gerader Gliederanzahl ausnimmt). Diese Reduktion der Positivitätsordnung der Reihe (26) um eine weitere Einheit, ist mir aber nur durch die unmittelbare Betrachtung der Reihe (26), und nicht durch die hier benutzte Methode der erzeugenden Funktion gelungen.

Da schliesslich

$$
F_{5}(r)=\left(\cos \theta-2 r+(\cos \theta) r^{2}\right)\left(1-r^{2}\right)^{-2}\left(F_{1}(r, \theta)\right)^{2},
$$

so ist

$$
(1-r)^{-4} F_{5}(r)=\left(\cos \theta-2 r+(\cos \theta) r^{2}\right)\left(1-r^{2}\right)^{-2}\left((1-r)^{-2} F_{1}(r, \theta)\right)^{2} .
$$

Für $\pi / 2<\theta<\pi$ sind die Koeffizienten des Polynoms zweiten Grades in $r$ : $\cos \theta-2 r+(\cos \theta) r^{2}$ negativ. Ich habe also erhalten, dass die Reihe

$$
\cos \theta+2 \cos 2 \theta+3 \cos 3 \theta+\cdots
$$

für $\pi / 2<\theta<\pi$ negativ von der dritten Ordnung ist.

III. PositivitÄt UND MONOTONITÄT VON TRIGONOMETRISCHEN REIHEN MIT EINFACH ODER MEHRFACH MONOTONEN KOEFFIZIENTENFOLGEN

1. Es sei

$$
\begin{aligned}
f(\theta) & =\alpha_{0} / 2+\sum_{n=1}^{\infty} \alpha_{n} \cos n \theta \\
& =\alpha_{0} / 2+\alpha_{1} \cos \theta+\alpha_{2} \cos 2 \theta+\cdots+\alpha_{n} \cos n \theta+\cdots
\end{aligned}
$$

eine unendliche trigonometrische Kosinusreihe mit nichtnegativen Koeffizienten. Ist ausserdem die Koeffizientenfolge monoton abnehmend und 
$\lim _{n \rightarrow \infty} \alpha_{n}=0$, d.h. ist $\left\{\alpha_{n}\right\}$ eine einfach monotone Nullfolge, so ist die Reihe (1), nach dem Schlömilchschen Satze, für $0<\theta<2 \pi$ überall konvergent, und in jedem Teilintervalle $\epsilon \leqq \theta \leqq 2 \pi-\epsilon, \epsilon>0$, gleichmässig konvergent. Die Summe der Reihe (1) sei für $0<\theta<2 \pi$ mit $f(\theta)$ bezeichnet; $f(\theta)$ ist stetig an jeder inneren Stelle des Intervalles $(0,2 \pi)$.

Ist nun die Folge $\left\{\alpha_{n}\right\}$ sogar eine zweifach monotone Nullfolge, d.h. ist

$$
\begin{aligned}
& \alpha_{0} \geqq 0, \\
& \alpha_{1} \geqq 0, \cdots, \\
& \alpha_{n} \geqq 0, \cdots, \\
& \alpha_{0}-\alpha_{1} \geqq 0, \\
& \alpha_{1}-\alpha_{2} \geqq 0, \cdots, \\
& \alpha_{n}-\alpha_{n+1} \geqq 0, \cdots, \\
& \alpha_{0}-2 \alpha_{1}+\alpha_{2} \geqq 0, \alpha_{1}-2 \alpha_{2}+\alpha_{3} \geqq 0, \cdots, \alpha_{n}-2 \alpha_{n+1}+\alpha_{n+2} \geqq 0, \cdots,
\end{aligned}
$$

so ist $f(\theta)$ im ganzen Intervalle $0<\theta<2 \pi$ nichtnegativ. Ist die erste der Differenzen zweiter Ordnung tatsächlich positiv, d.h. ist

$$
\alpha_{0}-2 \alpha_{1}+\alpha_{2}>0 \text {, }
$$

so ist $f(\theta)$ im ganzen Intervalle sogar positiv, und es gilt

$$
f(\theta) \geqq \frac{\alpha_{0}+\alpha_{2}}{2}-\alpha_{1} \text { für } 0<\theta<2 \pi .
$$

Dass dieser Satz bei einfach monotonen $\alpha_{n}$ nicht gültig ist, zeigt das triviale Beispiel

$$
f(\theta)=\frac{1}{2}+\cos \theta .
$$

Hier ist die Koeffizientenfolge $1,1,0,0,0, \ldots$ einfach monoton, während $f(\theta)$ für $2 \pi / 3<\theta<4 \pi / 3$ negativ ausfällt.

2. Ist $\alpha_{1}, \alpha_{2}, \alpha_{3}, \cdots$ eine vierfach monotone Nullfolge, d.h. ist

$$
\begin{aligned}
\alpha_{n} & \geqq 0, \quad \Delta^{1} \alpha_{n}=\alpha_{n}-\alpha_{n+1} \geqq 0, \quad \Delta^{2} \alpha_{n}=\alpha_{n}-2 \alpha_{n+1}+\alpha_{n+2} \geqq 0, \\
\Delta^{3} \alpha_{n} & =\alpha_{n}-3 \alpha_{n+1}+3 \alpha_{n+2}-\alpha_{n+3} \geqq 0, \\
\Delta^{4} \alpha_{n} & =\alpha_{n}-4 \alpha_{n+1}+6 \alpha_{n+2}-4 \alpha_{n+3}+\alpha_{n+4} \geqq 0
\end{aligned}
$$

für $n=1,2,3, \cdots$, und $\lim _{n \rightarrow \infty} \alpha_{n}=0$, so ist die Funktion $f(\theta)$, welche durch die für $0<\theta<2 \pi$ konvergente Reihe

$$
f(\theta)=\alpha_{0} / 2+\alpha_{1} \cos \theta+\alpha_{2} \cos 2 \theta+\cdots+\alpha_{n} \cos n \theta+\cdots
$$

definiert ist, monoton abnehmend im ganzen Intervalle $0<\theta<\pi$.

Beweis. Betrachten wir die für $0 \leqq r<1$ gewiss konvergente Reihe

$$
F(r, \theta)=\alpha_{0} / 2+\alpha_{1} r \cos \theta+\alpha_{2} r^{2} \cos 2 \theta+\cdots+\alpha_{n} r^{n} \cos n \theta+\cdots,
$$

sowie die Reihe 
(8) $\quad-\frac{d F(r, \theta)}{d \theta}=\alpha_{1} r \cdot \sin \theta+\alpha_{2} r^{2} \cdot 2 \sin 2 \theta+\cdots+\alpha_{n} r^{n} \cdot n \sin n \theta+\cdots$.

Bezeichnen wir nun die Summen dritter Ordnung der Reihe

$$
\sin \theta+2 \sin 2 \theta+\cdots+n \sin n \theta+\cdots
$$

mit

$$
s_{1}^{(3)}(\theta), s_{2}^{(3)}(\theta), s_{3}^{(3)}(\theta), \cdots, s_{n}^{(3)}(\theta), \cdots,
$$

so liefert eine viermalige Abelsche Umformung:

$$
\begin{aligned}
& \alpha_{1} r \cdot \sin \theta+\alpha_{2} r^{2} \cdot 2 \sin 2 \theta+\cdots+\alpha_{n} r^{n} \cdot n \sin n \theta \\
& =\sum_{\nu=1}^{n-4}\left(\alpha_{\nu} r^{\nu}-4 \alpha_{\nu+1} r^{\nu+1}+6 \alpha_{\nu+2} r^{\nu+2}-4 \alpha_{\nu+3} r^{\nu+3}+\alpha_{\nu+4} r^{\nu+4}\right) s_{\nu}^{(3)}(\theta) \\
& \quad+\left(\alpha_{n-3} r^{n-3}-4 \alpha_{n-2} r^{n-2}+6 \alpha_{n-1} r^{n-1}-4 \alpha_{n} r^{n}\right) s_{n-3}^{(3)}(\theta) \\
& \quad+\left(\alpha_{n-2} r^{n-2}-4 \alpha_{n-1} r^{n-1}+6 \alpha_{n} r^{n}\right) s_{n-2}^{(3)}(\theta) \\
& \quad+\left(\alpha_{n-1} r^{n-1}-4 \alpha_{n} r^{n}\right) s_{n-1}^{(3)}(\theta) \\
& \quad+\alpha_{n} r^{n} \cdot s_{n}^{(3)}(\theta) .
\end{aligned}
$$

Die roheste Abschätzung bei der Reihe (9) liefert $\left|s_{n}^{(3)}(\theta)\right| \leqq n^{5}$; da ferner $n^{5} r^{n} \rightarrow 0$, wenn $n \rightarrow \infty$, so folgt aus (11), durch den Grenzübergang $n \rightarrow \infty$,

$$
\begin{aligned}
-\frac{d F(r, \theta)}{d \theta} & =\sum_{\nu=1}^{\infty} \alpha_{\nu} \gamma^{\nu} \cdot \nu \sin \nu \theta \\
& =\sum_{\nu=1}^{\infty} s_{\nu}^{\left({ }^{3}\right)}(\theta) \Delta^{4}\left(\alpha_{\nu} \gamma^{\nu}\right) .
\end{aligned}
$$

Da aber $\alpha_{1}, \alpha_{2}, \ldots$ vierfach monoton, und $r, r^{2}, \ldots$ für $0 \leqq r<1$ vollmonoton ist, so ist $\alpha_{1} r, \alpha_{2} r^{2}, \cdots$ sicher vierfach monoton. Die vierten Differenzen $\Delta^{4}\left(\alpha_{\nu} \gamma^{\nu}\right), \nu=1,2,3, \cdots$, sind also durchweg nichtnegativ. Die Summen dritter Ordnung $s_{\nu}^{(3)}(\theta)$ der Reihe (9) sind nun für $0<\theta<\pi$ ebenfalls nichtnegativ, wie wir dies aus §II, Nr. 4, wissen. Also ist jedes Glied der konvergenten Reihe $\sum_{\nu=1}^{\infty} s_{\nu}^{(3)}(\theta) \Delta^{4}\left(\alpha_{\nu} r^{\nu}\right)$ nichtnegativ. Wir haben also

$$
\frac{d F(r, \theta)}{d \theta} \leqq 0 \text { für } 0<\theta<\pi
$$

erhalten; d.h. die Funktion $F(r, \theta)$ ist, bei festem $0 \leqq r<1$, im Intervalle $0<\theta<\pi$ monoton fallend. Da schiesslich nach dem Abelschen Grenzwertsatze

$$
f(\theta)=\lim _{r \rightarrow 1-0} F(r, \theta), \quad 0<\theta<\pi,
$$


so ist auch $f(\theta)$, als Grenzfunktion von monoton fallenden Funktionen, monoton fallend. Hiermit ist der angekündigte Satz bewiesen.*

1 te Bemerkung. Wir haben aus der vierfachen Monotonie der Koeffizienten $\alpha_{1}, \alpha_{2}, \alpha_{3}, \cdots$ geschlossen, dass $f(\theta)$ im Intervalle $(0, \pi)$ monoton fallend ist. Dieser Satz ist nicht richtig, wenn die Folge $\alpha_{1}, \alpha_{2}, \alpha_{3}, \cdots$ nur dreifach monoton ist. Z.B. hat die Reihe

$$
f(\theta)=3 \cos \theta+\cos 2 \theta
$$

die dreifach monotone Koeffizientenfolge $3,1,0,0,0, \ldots$. Es ist aber

$$
f^{\prime}(\theta)=-3 \sin \theta-2 \sin 2 \theta=-\sin \theta(3+4 \cos \theta),
$$

so dass also $f(\theta)$ in einem Teile des Intervalles $(0, \pi)$ monoton wächst. (Hingegen ist die Koeffizientenfolge in $f(\theta)=4 \cos \theta+\cos 2 \theta$ vierfach monoton und es gilt tatsächlich $f^{\prime}(\theta)=-\sin \theta(4+4 \cos \theta)$ für $0<\theta<\pi$.)

2te Bemerkung. Aus dem obigen Satze folgt, dass die Gleichung

$$
\sum_{\nu=1}^{\infty} \alpha_{\nu} \cos \nu \theta=0
$$

im Intervalle $0<\theta<\pi$ nur eine einzige Wurzel hat, falls $\left\{\alpha_{\nu}\right\}$ eine vierfach monotone Nullfolge ist. Ist $\left\{\alpha_{v}\right\}$ nur eine einfach monotone Nullfolge, so zeigt das triviale Beispiel

$$
\frac{\sin (n \theta / 2) \cos ((n+1) \theta / 2)}{\sin (\theta / 2)}=\cos \theta+\cos 2 \theta+\cdots+\cos n \theta=0
$$

dass die Gleichung beliebig viele Wurzeln im Intervalle $0<\theta<\pi$ haben kann.

3te Bemerkung. Ist $\alpha_{1}, \alpha_{2}, \alpha_{3}, \ldots$ vierfach monoton und $\alpha_{0} / 2, \alpha_{1}$, $\alpha_{2}, \alpha_{3}, \cdots$ mindestens zweifach monoton [was stets der Fall ist, wenn $\alpha_{0} \geqq 2\left(2 \alpha_{1}-\alpha_{2}\right)$ ], so ist $f(\theta)$, nach dem Vorhergehenden, für $0<\theta<\pi$ positiv und monoton fallend. Ist speziell schon $\alpha_{0}, \alpha_{1}, \alpha_{2}, \cdots$ zweifach monoton, ja

* Wir setzen immer $\alpha_{1} \geqq 0, \alpha_{2} \geqq 0, \cdots, \alpha_{n} \geqq 0, \cdots$ voraus, jedoch $\alpha_{1}=\alpha_{2}=\cdots=\alpha_{n}=\cdots=0$ sei stets ausgeschlossen. Dann folgt aber, wegen $\alpha_{n} \rightarrow 0$, dass mindestens ein Index $\mu$ existiert, so dass $\Delta^{4} \alpha_{\mu}>0$. Nun liefert (12)

und es ist nach Fussnote* auf S. 28

$$
-\frac{d F(r, \theta)}{d \theta} \geqq s_{\mu}{ }^{(3)}(\theta) \Delta^{4}\left(\alpha_{\mu} r^{\mu}\right)
$$

so dass

$$
\Delta^{4}\left(\alpha_{\mu} r^{\mu}\right) \geqq\left(\Delta^{4} \alpha_{\mu}\right) r^{\mu}
$$

$$
-\frac{d F(r, \theta)}{d \theta} \geqq\left(\Delta^{4} \alpha_{\mu}\right) r^{\mu} s_{\mu}{ }^{(3)}(\theta) \text {. }
$$

Die rechte Seite dieser Ungleichung konvergiert für $r \rightarrow 1$ gegen $\left(\Delta^{4} \alpha_{\mu}\right) s_{\mu}^{(3)}(\theta)$. Nun sind aber, wie wir in §II, Nr. 4, gesehen haben, die $s_{n}^{(3)}(\theta)$ der Reihe (9) im Intervalle $0<\theta<\pi$ durchweg positiv; sie haben ferner ebenda eine gemeinsame positive Minorante. Daraus schliesst man leicht, dass die Grenzfunktion $f(\theta)$ im Innern des Intervalles $(0, \pi)$ sogar eine eigentlich fallende Funktion von $\theta$ sein muss [d.h. $f\left(\theta_{1}\right)>f\left(\theta_{2}\right)$, wenn $\left.0<\theta_{1}<\theta_{2} \leqq \pi\right]$. 
sogar vierfach monoton, so ist also sicher $\sum_{\nu=0}^{\infty} \alpha_{\nu} \cos \nu \theta$ positiv und fallend im Intervalle $0<\theta<\pi$.

\section{Sind die Koeffizienten $\beta_{n}$ der trigonometrischen Sinusreihe}

$$
f(\theta)=\beta_{1} \sin \theta+\beta_{2} \sin 2 \theta+\cdots+\beta_{n} \sin n \theta+\cdots
$$

zweifach monoton, so ist sie für $0<\theta<\pi$ konvergent und ihre Summe $f(\theta)$ für $0<\theta<\pi$ positiv.

Ist die Folge $\left\{\beta_{n}\right\}$ sogar vierfach monoton, so ist $f(\theta)$, auf Grund von $§ I I$, im Intervalle $\pi / 2<\theta<\pi$ monoton fallend.

Beispiel. $f(\theta)=4 \sin \theta+\sin 2 \theta=\sin \theta(4+2 \cos \theta)$ hat eine vierfach monotone Koeffizientenfolge. Man sieht, dass $f(\theta)>0$ für $0<\theta<\pi$. Wegen $f^{\prime}(\theta)=4 \cos ^{2} \theta+4 \cos \theta-2$ ist tatsächlich $f^{\prime}(\theta)<0$ für $\pi / 2<\theta<\pi$. Wir sehen aber daraus weiter, dass $f(\theta)$ im Intervalle $(0, \pi / 2)$ teils zunimmt teils abnimmt.

\section{Ist die Koeffizientenfolge der trigonometrischen Kosinusreihe}

$$
f(\theta)=\sum_{\nu=1}^{\infty} \alpha_{\nu} \cos (2 \nu-1) \theta
$$

dreifach monoton, so ist ihre Summe $f(\theta)$ für $0<\theta<\pi / 2$ positiv, für $\pi / 2<\theta<\pi$ negativ, und im ganzen Intervalle $0<\theta<\pi$ monoton fallend. ${ }^{*}$

Die Koeffizientenfolge der Reihe $f(\theta)=2 \cos \theta+\cos 3 \theta=\cos \theta\left(4 \cos ^{2} \theta-1\right)$ ist zweifach monoton. Sie nimmt im Intervalle $0<\theta<\pi / 2$ tatsächlich auch negative Werte an. Hingegen ist $3 \cos \theta+\cos 3 \theta=4 \cos ^{3} \theta$ mit dreifach monotoner Koeffizientenfolge positiv für $0<\theta<\pi / 2$ und monoton abnehmend für $0<\theta<\pi$.

\section{Ist die Koeffizientenfolge der trigonometrischen Sinusreihe}

$$
f(\theta)=\sum_{\nu=1}^{\infty} \alpha_{\nu} \sin (2 \nu-1) \theta
$$

einfach monoton, so ist $f(\theta)$ für $0<\theta<\pi$ nichtnegativ.

Ist die erste der Differenzen $\alpha_{1}-\alpha_{2}, \alpha_{2}-\alpha_{3}, \cdots$ positiv, d.h. $\alpha_{1}-\alpha_{2}>0$, so ist $f(\theta)$ für $0<\theta<\pi$ sogar positiv und $\geqq\left(\alpha_{1}-\alpha_{2}\right) \sin \theta$.

6. Es sei

$$
f(\theta)=\sum_{\nu=0}^{\infty} c_{\nu} \sin (\nu+1) \theta=c_{0} \sin \theta+c_{1} \sin 2 \theta+c_{2} \sin 3 \theta+\cdots
$$

eine beliebige Sinusreihe mit einfach monotoner Koeffizientenfolge $\dagger c_{0}, c_{1}, c_{2}, \cdots$, $\lim _{n \rightarrow \infty} c_{n}=0$. Es bezeichne ferner a eine beliebig kleine, aber feste positive Zahl. Dann gibt es im Intervalle $(0, a)$ Stellen, an denen $f(\theta)$ positiv ist.

* So wie ihr erstes Glied $\alpha_{1} \cos \theta$.

† Die Reihe (19) braucht bekanntlich keine Fouriersche Reihe zu sein. 
Beweis. Da

$$
\begin{aligned}
f(\theta) & =\sum_{\nu=0}^{\infty} c_{\nu} \sin (\nu+1) \theta=\sum_{\nu=0}^{\infty} c_{\nu} \sin \left(\left(\nu+\frac{1}{2}\right) \theta+\theta / 2\right) \\
& =\left(\sum_{\nu=0}^{\infty} c_{\nu} \sin \left(\nu+\frac{1}{2}\right) \theta\right) \cos (\theta / 2)+\left(\sum_{\nu=0}^{\infty} c_{\nu} \cos \left(\nu+\frac{1}{2}\right) \theta\right) \sin (\theta / 2),
\end{aligned}
$$

so ist

$$
2 \sin (\theta / 2) f(\theta)=A(\theta) \cos (\theta / 2)+B(\theta) \sin (\theta / 2),
$$

wo

und

$$
\begin{aligned}
A(\theta) & =\sum_{\nu=0}^{\infty}\left(c_{\nu} \cdot 2 \sin \left(\nu+\frac{1}{2}\right) \theta \cdot \sin (\theta / 2)\right) \\
& =c_{0}-\sum_{\nu=1}^{\infty}\left(c_{\nu-1}-c_{\nu}\right) \cos \nu \theta,
\end{aligned}
$$

$$
\begin{aligned}
B(\theta) & =\sum_{\nu=0}^{\infty}\left(c_{\nu} \cdot 2 \cos \left(\nu+\frac{1}{2}\right) \theta \cdot \sin (\theta / 2)\right) \\
& =\sum_{\nu=1}^{\infty}\left(c_{\nu-1}-c_{\nu}\right) \sin \nu \theta .
\end{aligned}
$$

Aus (22) ersieht man, da $\left\{c_{\nu}\right\}$ eine monotone Nullfolge ist, dass

$$
A(\theta) \geqq c_{0}-\sum_{\nu=1}^{\infty}\left(c_{\nu-1}-c_{\nu}\right)=0
$$

für jeden Wert von $\theta$. Ferner sind $\cos (\theta / 2)$ und $\sin (\theta / 2)$ im Innern des Intervalles $(0, \pi)$ positiv. Kann ich also zeigen, dass

$$
B(\theta)=\sum_{\nu=1}^{\infty}\left(c_{\nu-1}-c_{\nu}\right) \sin \nu \theta
$$

im Intervalle $(0, a)$, wo $0<a<\pi$, auch einen positiven Wert annimmt, so ist, mit Rücksicht auf (21), unser Satz bewiesen. [Ich habe für die Reihe (25) dieselbe Behauptung zu beweisen, die ich für die Reihe (19) aufgestellt habe.] Für die Sinusreihe $B(\theta)$ unter (23) besteht aber die Koeffizientenreihe

$$
\sum_{\nu=1}^{\infty}\left(c_{\nu-1}-c_{\nu}\right)
$$

aus lauter nichtnegativen Gliedern und ist konvergent. Also ist die Sinusreihe von $B(\theta)$ für jedes $\theta$ gleichmässig konvergent. Die Integration von 0 bis $a$ liefert 


$$
\int_{0}^{a} B(\theta) d \theta=\sum_{\nu=1}^{\infty}\left(c_{\nu-1}-c_{\nu}\right) \frac{1-\cos \nu a}{\nu} .
$$

Wäre nun stets $B(\theta) \leqq 0$ im Intervalle $0<\theta<a$, so müsste

$$
\sum_{\nu=1}^{\infty}\left(c_{\nu-1}-c_{\nu}\right) \frac{1-\cos \nu a}{\nu} \leqq 0
$$

sein. Bezeichnet $p$ den ersten Index, für welchen in der Folge der nichtnegativen Differenzen

$$
c_{0}-c_{1}, c_{1}-c_{2}, c_{2}-c_{3}, \cdots
$$

zum erstenmal eine tatsächlich positive auftritt, so müsste also

$$
\left(c_{p-1}-c_{p}\right)(1-\cos p a) \leqq 0
$$

sein. Dies ist aber sicher nicht der Fall, wenn $0<a<\pi /(4 p)$, da dann die linke Seite von (30) positiv ausfällt.*

7. Übrigens ist der entsprechende Satz für die Kosinusreihe

$$
f(\theta)=c_{1} \cos \theta+c_{2} \cos 2 \theta+c_{3} \cos 3 \theta+\cdots
$$

mit einfach monotoner Koeffizientenfolge

$$
c_{1}, c_{2}, c_{3}, \cdots, \quad \lim _{n \rightarrow \infty} c_{n}=0,
$$

ebenfalls richtig. Da nämlich $\left\{c_{n}\right\}$ einfach monoton ist, so ist bekanntlich

$$
\sum_{n=1}^{\infty} c_{n} \frac{\sin n \theta}{n}
$$

für jedes $\theta$ gleichmässig konvergent. Es ist also, wenn $0<\theta \leqq a$,

$$
\int_{\theta}^{a} f(t) d t=\sum_{n=1}^{\infty} c_{n} \frac{\sin n a}{n}-\sum_{n=1}^{\infty} c_{n} \frac{\sin n \theta}{n}
$$

so dass für $\theta \rightarrow+0$

$$
\int_{0}^{a} f(t) d t=\sum_{n=1}^{\infty} c_{n} \frac{\sin n a}{n}
$$

Nun ist bekanntlich

$$
\frac{\sin a}{1}+\frac{\sin 2 a}{2}+\cdots+\frac{\sin n a}{n}>0
$$

* Úbrigens zeigen die Formeln (21), (22), (23) auch, dasss $(\sin \theta) f(\theta)$ für $\theta \rightarrow+0$ zu 0 strebt, während $f(\theta)$ für $\theta \rightarrow+0$ auf eine nicht integrable Weise unendlich werden kann. 
für $0<a<\pi$, und für jedes $n$. Wenn also $\left\{c_{n}\right\}$ eine nichtnegative einfach monotone Nullfolge bezeichnet, so ist auch $\sum_{n=1}^{\infty} c_{n}(\sin n a) / n$ positiv im Intervalle $0<a<\pi$. Also ist nach (35)

$$
\int_{0}^{a} f(t) d t>0
$$

Es ist somit unmöglich, dass für die Kosinusreihe $f(\theta)$ unter (31) im Intervalle $0<\theta \leqq a$ beständig $f(\theta) \leqq 0$ gültig wäre. Hier bezeichnet $a$ eine positive Zahl.

8. Mit Rücksicht auf die Anwendung auf die Sinusreihe des Legendreschen Polynoms $P_{n}(\cos \theta)$ erwähne ich den folgenden, dem vorigen entsprechenden Satz:

Besitzt die Sinusreihe

$$
f(\theta)=\sum_{\nu=0}^{\infty} c_{\nu} \sin (n+2 \nu+1) \theta=c_{0} \sin (n+1) \theta+c_{1} \sin (n+3) \theta+\cdots
$$

eine einfach monotone Koeffizientenfolge

$$
c_{0}, c_{1}, c_{2}, \cdots,
$$

so nimmt ihre Summe $f(\theta)$ in jedem noch so kleinen Intervalle $(0, a)$, wo $a>0$, positive Werte an.

Der Beweis verläuft dem in Nr. 6 gegebenen ganz ähnlich. Er beruht jetzt auf der Gleichung

$$
2(\sin \theta) f(\theta)=A(\theta) \cos n \theta+B(\theta) \sin n \theta,
$$

wo

$$
\begin{aligned}
& A(\theta)=c_{0}-\sum_{\nu=1}^{\infty}\left(c_{\nu-1}-c_{\nu}\right) \cos 2 \nu \theta, \\
& B(\theta)=\sum_{\nu=1}^{\infty}\left(c_{\nu-1}-c_{\nu}\right) \sin 2 \nu \theta .
\end{aligned}
$$

IV. ÚbER dIE NULLSTELLEN VON TRIGONOMETRISCHEN RESTREIHEN MIT EINFACH ODER MEHRFACH MONOTONEN KOEFFIZIENTEN.

\section{HEINESCHER TyPUS}

1. Ein Polynom oder eine Reihe von der Form

$$
\begin{aligned}
& a_{n+1} \cos (n+1) \theta+a_{n+2} \cos (n+2) \theta+\cdots, \\
& b_{n+1} \sin (n+1) \theta+b_{n+2} \sin (n+2) \theta+\cdots
\end{aligned}
$$

möge eine trigonometrische Restreihe heissen. In Anbetracht der Anwendung 
auf die Legendreschen Polynome möchte ich hier besonders ausführlich die Sinusreihe behandeln, und auch diese nur im Falle, wo bloss der Sinus eines jeden zweiten Multiplums von $\theta$ in der Reihe tatsächlich auftritt. Bei veränderter Bezeichnung handelt es sich also um die Reihe

(3) $f(\theta)=\sum_{\nu=0}^{\infty} c_{\nu} \sin (n+2 \nu+1) \theta=c_{0} \sin (n+1) \theta+c_{1} \sin (n+3) \theta+\cdots$,

wo $n$ eine feste nichtnegative ganze Zahl bedeutet. Von einer trigonometrischen Reihe von dieser Form sage ich, dass sie vom Heineschen Typus ist.

Die Koeffizientenfolge $c_{0}, c_{1}, \cdots, c_{\nu}, \cdots$ sei mindestens einfach monoton, ferner sei mindestens $c_{0}-c_{1}>0$. Weiter möge $\lim _{\nu \rightarrow \infty} c_{\nu}=0$ sein. Dann konvergiert die Reihe (3) für jeden Wert $0 \leqq \theta \leqq \pi$ und ist in jedem Teilintervalle $\epsilon \leqq \theta \leqq \pi-\epsilon, \epsilon>0$, gleichmässig konvergent. Ihre mit $f(\theta)$ bezeichnete Summe ist also für jedes $0<\theta<\pi$ stetig.

Ich zerlege nun die Reihe, ähnlich wie es Szegö im Falle eines trigonometrischen Polynoms tat, in zwei Summanden, und zwar hier in dreifacher Weise:

$$
\begin{aligned}
& f(\theta)=p(\theta) \sin (n-1) \theta+q(\theta) \cos (n-1) \theta, \\
& f(\theta)=r(\theta) \sin n \theta+s(\theta) \cos n \theta, \\
& f(\theta)=t(\theta) \sin (n+1) \theta+u(\theta) \cos (n+1) \theta,
\end{aligned}
$$

wo

$$
\begin{aligned}
p(\theta) & =\sum_{\nu=0}^{\infty} c_{\nu} \cos 2(\nu+1) \theta, & q(\theta) & =\sum_{\nu=0}^{\infty} c_{\nu} \sin 2(\nu+1) \theta, \\
r(\theta) & =\sum_{\nu=0}^{\infty} c_{\nu} \cos (2 \nu+1) \theta, & s(\theta) & =\sum_{\nu=0}^{\infty} c_{\nu} \sin (2 \nu+1) \theta, \\
t(\theta) & =\sum_{\nu=0}^{\infty} c_{\nu} \cos 2 \nu \theta, & u(\theta) & =\sum_{\nu=0}^{\infty} c_{\nu} \sin 2 \nu \theta .
\end{aligned}
$$

Die sechs trigonometrischen Reihen $p(\theta), \cdots, u(\theta)$ sind im Intervalle $0<\theta<\pi$ überall konvergent, im Intervalle $\epsilon \leqq \theta \leqq \pi-\epsilon$ ferner gleichmässig konvergent, so dass die sechs Funktionen $p(\theta), \cdots, u(\theta)$ im Intervalle $0<\theta<\pi$ sämtlich stetig sind. Je nachdem man die Wurzeln der sechs Gleichungen

$$
\begin{aligned}
\sin (n-1) \theta & =0, & \cos (n-1) \theta & =0, \\
\sin n \theta & =0, & \cos n \theta & =0, \\
\sin (n+1) \theta & =0, & \cos (n+1) \theta & =0
\end{aligned}
$$


in Betracht zieht und eventuell die Koeffizientenfolge noch der Bedingung einer höheren Monotonie unterwirft, erhält man verschiedene Aussagen über die Wurzeln der Gleichung $f(\theta)=0$ im Intervalle $0<\theta<\pi$. Ich werde von diesen 6 Fällen nur die 3 behandeln, die ich für wichtiger halte.

2. 1 ter Fall: $\sin n \theta=0$, d.h.

$$
\theta_{m}=m \pi / n \quad(m=1,2, \cdots,(n-1)) .
$$

Das sind diejenigen Wurzeln von $\sin n \theta=0$, die in das Innere des Intervalles $(0, \pi)$ fallen. Die Gleichung (5) liefert num

$$
f\left(\theta_{m}\right)=s\left(\theta_{m}\right) \cos n \theta_{m}=s\left(\theta_{m}\right) \cos m \pi=s\left(\theta_{m}\right)(-1)^{m} .
$$

Da aber $s(\theta)$ unter (8), wie in §III gezeigt wurde, im Intervalle $0<\theta<\pi$ überall positiv ist, so ist

$$
\operatorname{sgn} f\left(\theta_{m}\right)=(-1)^{m} \quad(m=1,2, \cdots,(n-1)) .
$$

Ich habe also erhalten, dass $f(\theta)$ in den $(n-2)$ Intervallen $(\pi / n, 2 \pi / n)$, $(2 \pi / n, 3 \pi / n), \cdots,((n-2) \pi / n,(n-1) \pi / n)$ je eine Nullstelle besitzt. Da aber $f\left(\theta_{1}\right)=f(\pi / n)$ negativ ist, und $f(\theta)$, nach dem Satz in §III, Nr. 8, in beliebiger rechtsseitiger Umgebung von $\theta=0$ auch positive Werte annimmt, so muss es auch im Innern des ersten Intervalles $(0, \pi / n)$ eine Nullstelle besitzen. Daraus folgt, auf Grund der aus (3) resultierenden Symmetriegleichung

$$
\begin{aligned}
f(\pi-\theta) & =\sum_{\nu=0}^{\infty} c_{\nu} \sin (n+2 \nu+1)(\pi-\theta) \\
& =-\sum_{\nu=0}^{n} c_{\nu} \cos (n+2 \nu+1) \pi \sin (n+2 \nu+1) \theta=(-1)^{n} f(\theta),
\end{aligned}
$$

dass $f(\theta)$ auch im Innern des letzten Intervalles $((n-1) \pi / n, \pi)$ eine Nullstelle hat. Es gilt somit der folgende Satz:

Sind $c_{0}, c_{1}, c_{2}, \cdots$ nicht negative, nicht zunehmende Zahlen mit $\lim _{\nu \rightarrow \infty} c_{\nu}=0$, so ist die Reihe

$$
f(\theta)=\sum_{\nu=0}^{\infty} c_{\nu} \sin (n+2 \nu+1) \theta=c_{0} \sin (n+1) \theta+c_{1} \sin (n+3) \theta+\cdots
$$

im Intervalle $0<\theta<\pi$ überall konvergent und ihre Summe $f(\theta)$ ist für diese Werte von $\theta$ eine stetige Funktion. Teill man das Intervall $(0, \pi)$ in $n$ gleiche Teile, so besitzt $f(\theta)$ in jedem dieser Teilintervalle mindestens eine Nullstelle (sogar eine Zeichenwechselstelle). D.h. $f(\theta)$ hat im Intervalle $(0, \pi)$ mindestens $n$ Nullstellen $t_{1}, t_{2}, \cdots, t_{n}$, so dass

$$
(k-1) \pi / n<t_{k}<k \pi / n \quad(k=1,2, \cdots, n) .
$$


3. 2ter Fall: $\sin (n+1) \theta=0$. Es sei also jetzt

$$
\theta_{m}=m \frac{\pi}{n+1} \quad\left(m=0,1,2, \cdots, n^{\prime}=\left[\frac{n+1}{2}\right]\right) .
$$

Wir erhalten durch Einsetzen in die Gleichung (6)

$$
f\left(\theta_{m}\right)=u\left(\theta_{m}\right) \cos m \pi=u\left(\theta_{m}\right)(-1)^{m} .
$$

Es sei nun $\left\{c_{\nu}\right\}$ eine zweifach monotone Nullfolge. Dann ist nach §III

$$
u(\theta)=\sum_{\nu=0}^{\infty} c_{\nu} \sin 2 \nu \theta
$$

positiv im Innern des Intervalles $(0, \pi / 2)$. Nun sei erstens $n$ gerade. Dann fallen $\theta_{1}, \theta_{2}, \cdots, \theta_{n^{\prime}}$, wo $n^{\prime}=n / 2$, in das Innere von $(0, \pi / 2)$, so dass also

$$
\operatorname{sgn} f\left(\theta_{m}\right)=(-1)^{m}
$$

besteht. Dies besagt, dass $f(\theta)$ im Innern jedes der Intervalle $\left(\theta_{1}, \theta_{2}\right)$, $\left(\theta_{2}, \theta_{3}\right), \cdots,\left(\theta_{n^{\prime}-1}, \theta_{n^{\prime}}\right)$ je eine Nullstelle, insgesamt also $n^{\prime}-1$ Nullstellen besitzt. Da aber auch im Innern von $\left(0, \theta_{1}\right)$ eine Nullstelle vorhanden ist, so haben wir also bewiesen, dass $f(\theta)$ im Innern von $(0, \pi / 2)$ insgesamt $n^{\prime}$, und infolge der Symmetrieeigenschaft (12) im Innern von $(0, \pi) 2 n^{\prime}=n$ voneinander verschiedene Nullstellen hat. Zweitens sei $n$ ungerade. Dann haben wir wieder $n^{\prime}-1$ Nullstellen im Innern von $(0, \pi / 2)$, also $2 n^{\prime}-2 \mathrm{im}$ Innern von $(0, \pi)$. Hierzu tritt jetzt noch die Nullstelle $\theta_{n^{\prime}}=\pi / 2$. Wir haben also im Innern von $(0, \pi)$ wieder $2 n^{\prime}-1=n$ Nullstellen. Ich habe also den folgenden Satz bewiesen:

Sind die Zahlen $\left\{c_{\nu}\right\}$ zweifach monoton und $\lim _{\nu \rightarrow \infty} c_{\nu}=0$, so hat die Summe $f(\theta)$ der Reihe

$$
f(\theta)=\sum_{\nu=0}^{\infty} c_{\nu} \sin (n+2 \nu+1) \theta
$$

im Intervalle $0<\theta<\pi$ mindestens $n$ Nullstellen $t_{1}, t_{2}, \cdots, t_{n}$ (die zur Mitte $\theta=\pi / 2$ symmetrisch gelegen sind). Es sind die Grenzen

$$
(m-1) \frac{\pi}{n+1}<t_{m}<m \frac{\pi}{n+1}
$$

gültig, wobei $m=1,2,3, \cdots, n^{\prime}=[(n+1) / 2]$.

Ist $n$ ungerade, so muss in der letzten Ungleichung $t_{n^{\prime}}<n^{\prime} \pi /(n+1)$ statt $<$ das Gleichheitszeichen geschrieben werden. 
4. 3ter Fall: $\cos n \theta=0$, also

$$
\theta_{m}^{*}=(2 m-1) \pi /(2 n) .
$$

Jetzt liefert die Gleichung (5)

$$
f\left(\theta_{m}^{*}\right)=r\left(\theta_{m}^{*}\right) \sin ((2 m-1) \pi / 2)=r\left(\theta_{m}^{*}\right)(-1)^{m+1} .
$$

Es sei die Nullfolge $\left\{c_{\nu}\right\}$ dreifach monoton. Dann ist

$$
r(\theta)=\sum_{\nu=0}^{\infty} c_{\nu} \cos (2 \nu+1) \theta
$$

im Innern von $(0, \pi / 2)$ positiv. Es ist also

$$
\operatorname{sgn} f\left(\theta_{m}{ }^{*}\right)=(-1)^{m+1},
$$

solange $\theta_{m}^{*}$ im Innern von $(0, \pi / 2)$ liegt.

5. Betrachten wir nun nochmals die $\theta$-Stellen des 2 ten Falles:

$$
\theta_{m}=m \frac{\pi}{n+1} .
$$

Wir können zunächst feststellen, dass

$$
\theta_{1}^{*}<\theta_{1}<\theta_{2}^{*}<\theta_{2}<\theta_{3}^{*}<\theta_{3}<\cdots<\theta_{n^{\prime}}^{*}<\theta_{n^{\prime}}<\pi / 2, n^{\prime}=\left[\frac{n+1}{2}\right] .
$$

Diese Ungleichungskette ist für gerade $n$ in der Form richtig, wie sie niedergeschrieben ist. Ist aber $n$ ungerade, so muss das Ende statt $\theta_{n^{\prime}}^{*}<\theta_{n^{\prime}}$ $<\pi / 2$ so heissen: $\theta_{n^{\prime}}^{*}=\theta_{n^{\prime}}=\pi / 2$.

Da nach (24) sgn $f\left(\theta_{m}^{*}\right)=(-1)^{m+1}$ und nach (18) $\operatorname{sgn} f\left(\theta_{m}\right)=(-1)^{m}$ ist, so hat $f(\theta)$ offenbar in jedem der $n^{\prime}=[(n+1) / 2]$ Intervalle $\left(\theta_{m}^{*}, \theta_{m}\right)$, $m=1,2, \cdots, n^{\prime}$, je eine Nullstelle. Diese Feststellung ist im Falle eines ungeraden $n$ nur insofern $z u$ modifizieren, als in diesem Falle für das letzte Paar $\theta_{n^{\prime}}^{*}=\theta_{n^{\prime}}=\pi / 2$ gültig ist, also das Intervall $\left(\theta_{n^{\prime}}^{*}, \theta_{n^{\prime}}\right)$ auf den Punkt $\pi / 2$ zusammenschrumpft. Wegen (3) ist aber jetzt $\theta=\pi / 2$ augenscheinlich eine Wurzel von $f(\theta)=0$. Ich habe also das folgende Theorem erhalten:

Ist die Koeffizientenfolge $\left\{c_{\nu}\right\}$ der trigonometrischen Reihe

$$
f(\theta)=\sum_{\nu=0}^{\infty} c_{\nu} \sin (n+2 \nu+1) \theta=c_{0} \sin (n+1) \theta+c_{1} \sin (n+3) \theta+\cdots
$$

eine dreifach monotone Nullfolge, so hat die für $0<\theta<\pi$ stetige Funktion $f(\theta)$ im Intervalle $(0, \pi)$ mindestens $n$ Nullstellen $t_{1}, t_{2}, \cdots, t_{n}$. Für die im $0<\theta \leqq \pi / 2$ gelegenen Nullstellen gelten die Schranken 


$$
\left(m-\frac{1}{2}\right) \frac{\pi}{n}<t_{m}<m \frac{\pi}{n+1} \quad\left(m=1,2,3, \cdots, n^{\prime}=\left[\frac{n+1}{2}\right]\right) .
$$

Ist $n$ ungerade, so muss statt der letzten dieser Ungleichungen

$$
\left(n^{\prime}-\frac{1}{2}\right) \frac{\pi}{n}=t_{n^{\prime}}=n^{\prime} \frac{\pi}{n+1}(=\pi / 2)
$$

geschricben werden.

V. ÜBER DIE NULLSTELLEN EINER BELIEBIGEN TRIGONOMETRISCHEN

RESTREIHE MIT EINFACH ODER MEHRFACH MONOTONEN

\section{KOEFFIZIENTEN}

1. Es sei nun eine trigonometrische Sinusreihe von der Form

$$
b_{n+1} \sin (n+1) \theta+b_{n+2} \sin (n+2) \theta+b_{n+3} \sin (n+3) \theta+\cdots
$$

vorgegeben, in welcher alle ganzzahligen Vielfachen von $\theta$, vom $(n+1)$ ten angefangen, vorkommen. Mit der entsprechenden Kosinusreihe will ich mich auch jetzt nicht beschäftigen. Ich setze mit abgeänderter Bezeichnung

$$
f(\theta)=\sum_{\nu=0}^{\infty} c_{\nu} \sin (n+\nu+1) \theta
$$

und betrachte jetzt die Zerlegungen

$$
\begin{aligned}
& f(\theta)=A(\theta) \sin n \theta+B(\theta) \cos n \theta, \\
& f(\theta)=C(\theta) \sin \left(n+\frac{1}{2}\right) \theta+D(\theta) \cos \left(n+\frac{1}{2}\right) \theta, \\
& f(\theta)=E(\theta) \sin (n+1) \theta+F(\theta) \cos (n+1) \theta,
\end{aligned}
$$

wo

$$
\begin{array}{lll}
A(\theta)=\sum_{\nu=0}^{\infty} c_{\nu} \cos (\nu+1) \theta, & B(\theta)=\sum_{\nu=0}^{\infty} c_{\nu} \sin (\nu+1) \theta, \\
C(\theta)=\sum_{\nu=0}^{\infty} c_{\nu} \cos \left(\nu+\frac{1}{2}\right) \theta, & D(\theta)=\sum_{\nu=0}^{\infty} c_{\nu} \sin \left(\nu+\frac{1}{2}\right) \theta, \\
E(\theta)=\sum_{\nu=0}^{\infty} c_{\nu} \cos \nu \theta, & F(\theta)=\sum_{\nu=0}^{\infty} c_{\nu} \sin \nu \theta .
\end{array}
$$

2. 1 ter Fall: $\sin \left(n+\frac{1}{2}\right) \theta=0$, also

$$
\theta_{m}^{(1)}=m \frac{\pi}{n+\frac{1}{2}}=\frac{2 m \pi}{2 n+1} \quad(m=1,2, \cdots, n) .
$$

Ist nun $c_{0}, c_{1}, \cdots, c_{\nu}, \cdots$ bloss einfach monoton, so sind $C(\theta)$ und $D(\theta)$ 
in $0<\theta<2 \pi$ konvergent und stetig und $D(\theta)$ für $0<\theta<\pi$ gewiss positiv. Also liefert die Gleichung (4)

$$
f\left(\theta_{m}^{(1)}\right)=D\left(\theta_{m}^{(1)}\right) \cos m \pi
$$

und

$$
\operatorname{sgn} f\left(\theta_{m}^{(1)}\right)=(-1)^{m} \quad(m=1,2, \cdots, n) .
$$

3. 2ter Fall: $\sin (n+1) \theta=0$, also

$$
\theta_{m}^{(2)}=m \frac{\pi}{n+1} \quad(m=1,2, \cdots, n) .
$$

Die Gleichung (5) liefert

$$
f\left(\theta_{m}^{(2)}\right)=F\left(\theta_{m}^{(2)}\right) \cos m \pi .
$$

Ist aber $c_{1}, c_{2}, c_{3}, \cdots, c_{v}, \cdots$ zweifach monoton, ${ }^{*}$ so wird

$$
F(\theta)=\sum_{\nu=0}^{\infty} c_{\nu} \sin \nu \theta=c_{1} \sin \theta+c_{2} \sin 2 \theta+\cdots
$$

positiv für $0<\theta<\pi$, also

$$
\operatorname{sgn} f\left(\theta_{m}^{(2)}\right)=(-1)^{m} \quad(m=1,2, \cdots, n) .
$$

4. 3ter Fall: $\cos \left(n+\frac{1}{2}\right) \theta=0$, also

$$
\theta_{m}^{(3)}=(2 m-1) \frac{\pi}{2 n+1} \quad(m=1,2, \cdots, n) .
$$

Ist $c_{0}, c_{1}, \cdots, c_{\nu}, \cdots$ dreifach monoton, so wird

$$
\begin{aligned}
C(\theta) & =\sum_{\nu=0}^{\infty} c_{\nu} \cos \left(\nu+\frac{1}{2}\right) \theta \\
& =c_{0} \cos (\theta / 2)+c_{1} \cos (3 \theta / 2)+c_{2} \cos (5 \theta / 2)+\cdots
\end{aligned}
$$

für $0<\theta<\pi$ positiv. Man hat also

$$
f\left(\theta_{m}^{(3)}\right)=C\left(\theta_{m}^{(3)}\right) \sin (2 m-1) \pi / 2,
$$

d.h.

$$
\operatorname{sgn} f\left(\theta_{m}^{(3)}\right)=(-1)^{m+1} \quad(m=1,2, \cdots, n) .
$$

5. Es ist

(20) $\theta_{1}{ }^{(3)}<\theta_{1}{ }^{(2)}<\theta_{2}{ }^{(3)}<\theta_{2}{ }^{(2)}<\theta_{3}^{(3)}<\theta_{3}{ }^{(2)}<\cdots<\theta_{n}{ }^{(3)}<\theta_{n}{ }^{(2)}$.

\footnotetext{
* Man beachte die Unabhängigkeit von $c_{0}$.
} 
Dies folgt z.B. unmittelbar aus dem Umstande, dass die $\theta_{m}{ }^{(8)}$ aufeinanderfolgende Wurzeln von $\cos \left(n+\frac{1}{2}\right) \theta=0$, die $\theta_{m}^{(2)}$ aber aufeinanderfolgende Wurzeln von $\sin (n+1) \theta=0$ sind, und dass

$$
\sin (n+1) \theta=\sin \left(n+\frac{1}{2}\right) \theta \cos (\theta / 2)+\cos \left(n+\frac{1}{2}\right) \theta \sin (\theta / 2) .
$$

Übrigens ist die Länge des Intervalles $\left(\theta_{m}^{(3)}, \theta_{m}^{(2)}\right)$

$$
\begin{aligned}
\theta_{m}^{(2)}-\theta_{m}^{(3)} & =m \frac{\pi}{n+1}-(2 m-1) \frac{\pi}{2 n+1} \\
& =\frac{n-m+1}{(n+1)(2 n+1)} \pi \\
& =\left(1-\frac{m}{n+1}\right) \frac{\pi}{2 n+1} \quad(m=1,2, \cdots, n) .
\end{aligned}
$$

Die Länge dieser getrennten Intervalle nimmt also in arithmetischer Progression ab, wenn der Reihe nach $m=1,2, \cdots, n$. Im Anfangspunkt $\theta_{m}{ }^{(8)}$ ist das Vorzeichen von $f(\theta)$ gleich $(-1)^{m+1}$, im Endpunkte $\theta_{m}^{(2)}$ gleich $(-1)^{m}$. Ich kann also auf Grund des Vorhergehenden den folgenden Satz aussprechen:

Bilden die Koeffizienten der Reihe

$$
f(\theta)=b_{n+1} \sin (n+1) \theta+b_{n+2} \sin (n+2) \theta+\cdots
$$

eine einfach monotone Nullfolge (d.h. eine gewöhnliche nichtnegative Nullfolge), dann hat die im Intervalle $0<\theta<\pi$ stetige Funktion $f(\theta)$ im Innern dieses Intervalles mindestens $n$ voneinander verschiedene Nullstellen, und zwar mindestens je eine im Innern der $n$ Intervalle

$$
\left((m-1) \frac{\pi}{n+\frac{1}{2}}, \quad m \frac{\pi}{n+\frac{1}{2}}\right) \quad(m=1,2, \cdots, n) .
$$

Dieser Satz lässt sich insofern nicht verschärfen, als eine Reihe von der Form (23) in besonderen Fällen genau $n$ Nullstellen im Innern von $(0, \pi)$ haben kann. Dies zeigt das triviale Beispiel

$$
\begin{aligned}
& b_{n+1}=1, \\
& b_{n+2}=b_{n+3}=\cdots=0,
\end{aligned}
$$

d.h. die Funktion sin $(n+1) \theta$. [Thre sämtl chen Nullstellen in $(0, \pi)$ sind $m \pi /(n+1), m=1,2, \cdots, n$. Sie liegen einzeln in den Intervallen (24).]

Auf Sinuspolynome angewendet, lautet dieser Satz folgendermassen: 
Ist die Koeffizientenfolge des Sinuspolynoms

(26) $f(\theta)=c_{n+1} \sin (n+1) \theta+c_{n+2} \sin (n+2) \theta+\cdots+c_{p} \sin p \theta$

monoton abnehmend und positiv, d.h.

$$
c_{n+1}>c_{n+2}>\cdots>c_{p}>0,
$$

so besitzt $f(\theta)$ im Innern des Intervalles $(0, \pi)$ mindestens $n$ Nullstellen $t_{1}, t_{2}, \cdots, t_{n}$, für welche die Ungleichungen

$$
(m-1) \frac{\pi}{n+\frac{1}{2}}<t_{m}<m \frac{\pi}{n+\frac{1}{2}} \quad(m=1,2, \cdots, n)
$$

gelten.

Hierzu und zum vorigen Satze über den Fall der unendlichen Reihe bemerke ich folgendes. Sturm hat bewiesen, dass ein Sinuspolynom (26) $\left(c_{n+1} \neq 0, c_{p} \neq 0\right)$ im Innern des Intervalles $(0, \pi)$ höchstens $p-1$, und mindestens $n$ Nullstellen hat, welche Werte auch seine Koeffizienten $c_{n+1}$, $c_{n+2}, \cdots, c_{p}$ haben mögen. Hurwitz [15] hat den Satz von Sturm verallgemeinert, indem er bewies, dass auch die unendliche Sinusreihe (1) im Innern des Intervalles $(0, \pi)$ mindestens $n$ Nullstellen besitzt, vorausgesetzt, dass sie die Fouriersche Sinusreihe einer im Intervalle $0 \leqq \theta \leqq \pi$ beschränkten und für $0<\theta<\pi$ stetigen Funktion ist.

Unser Satz über das Sinuspolynom und über die unendliche Sinusreihe enthält nun eine Präzisierung der Sätze von Sturm und Hurwitz in Szegöscher Richtung, insofern, als er $n$ getrennte Teilintervalle in $(0, \pi) \mathrm{mit}$ mindestens je einer Nullstelle der endlichen oder unendlichen Reihe liefert, allerdings für den speziellen, jedoch nicht unwichtigen Fall, in welchem die Koeffizienten positiv und monoton abnehmend sind. (Was den Fall der unendlichen Reihe anbelangt, so sei bemerkt, dass mein Satz z.B. auch die Reihe

$$
\frac{\sin (n+1) \theta}{\log (n+1)}+\frac{\sin (n+2) \theta}{\log (n+2)}+\cdots
$$

erfasst, die bekanntlich keine Fouriersche Reihe ist.)

6. Auf Grund des Vorhergehenden gilt auch der folgende Satz:

Ist die Koeffizientenfolge der unendlichen trigonometrischen Reihe

$$
f(\theta)=b_{n+1} \sin (n+1) \theta+b_{n+2} \sin (n+2) \theta+\cdots
$$

eine dreifach monotone Nullfolge, so hat ihre für $0<\theta<\pi$ stetige Summe $f(\theta)$ in jeder der folgenden $n$, vollständig getrennten, und von den Werten der Koeffzienten unabhängigen Intervallen 


$$
\left((2 m-1) \frac{\pi}{2 n+1}, m \frac{\pi}{n+1}\right) \quad(m=1,2, \cdots, n)
$$

mindestens eine Nullstelle.

(Die Länge des $m$ ten Intervalles ist

$$
\frac{n-m+1}{(n+1)(2 n+1)} \pi
$$

und die Summe der $n$ Intervalle ist $n \pi /(4 n+2)$, so dass also das Verhältnis der Gesamtlänge der Einschränkungsintervalle (31) zu $\pi$ gegen $\frac{1}{4}$ konvergiert, wenn $n \rightarrow \infty$.)

VI. Die HeINESChEN UNENDLICHEN TRIgonometrischen REIHEN FÜr DIE KUgELFUNKTIONEN. ANWENDUNG DER DARGELEGTEN ALLGEMEINEN Resultate auf diesen Spezialfall

1. Heine hat für die Fouriersche Sinusreihe des $n$ ten Legendreschen Polynoms die folgende Form gefunden [Heine 13 Bd. I, S. 89, Stieltjes 24, Hobson 14]:

$$
\begin{aligned}
(\pi / 4) P_{n}(\cos \theta)= & \frac{2 \cdot 4 \cdots 2 n}{3 \cdot 5 \cdots(2 n+1)}\left\{\sin (n+1) \theta+\frac{1}{1} \frac{n+1}{2 n+3} \sin (n+3) \theta\right. \\
& \left.+\frac{1 \cdot 3}{1 \cdot 2} \frac{(n+1)(n+2)}{(2 n+3)(2 n+5)} \sin (n+5) \theta+\cdots\right\},
\end{aligned}
$$

d.h.

(2) $(\pi / 4) P_{n}(\cos \theta)=\frac{2 \cdot 4 \cdots 2 n}{3 \cdot 5 \cdots(2 n+1)} \sum_{\nu=0}^{\infty} h_{\nu} \sin (n+2 \nu+1) \theta, \quad 0<\theta<\pi$, wo

$$
h_{0}=1, \quad h_{\nu}=\prod_{k=1}^{\nu} \frac{2 k-1}{k} \frac{n+k}{2 n+2 k+1} \quad(\nu=1,2,3, \cdots) .
$$

Führt man die Koeffizienten $\alpha_{\nu}$ ein, definiert durch

$$
(1-z)^{-1 / 2}=\sum_{\nu=0}^{\infty} \alpha_{\nu} z^{\nu} ; \quad \alpha_{0}=1, \quad \alpha_{\nu}=\frac{1 \cdot 3 \cdot 5 \cdots(2 \nu-1)}{2 \cdot 4 \cdot 6 \cdots 2 \nu},
$$

so findet man, dass auch

$$
(\pi / 4) \alpha_{n}(2 n+1) P_{n}(\cos \theta)
$$

$$
=\sum_{\nu=0}^{\infty} \alpha_{\nu} \frac{(2 n+2)(2 n+4) \cdots(2 n+2 \nu)}{(2 n+3)(2 n+5) \cdots(2 n+2 \nu+1)} \sin (n+2 \nu+! 1) \theta,
$$


d.h.

$$
\begin{aligned}
(\pi / 4) P_{n}(\cos \theta) & =\sum_{\nu=0}^{\infty} \frac{\alpha_{\nu}}{\alpha_{n+\nu}} \frac{\sin (n+2 \nu+1) \theta}{2 n+2 \nu+1} \\
& =\sum_{\nu=0}^{\infty} c_{\nu} \sin (n+2 \nu+1) \theta, \quad 0<\theta<\pi,
\end{aligned}
$$

gültig ist, wobei*

$$
\begin{aligned}
c_{\nu}^{(n)}=c_{\nu}=\frac{(2 \nu+2)(2 \nu+4) \cdots(2 \nu+2 n)}{(2 \nu+1)(2 \nu+3) \cdots(2 \nu+2 n-1)} \frac{1}{2 \nu+2 n+1} & \\
(\nu & =0,1,2,3, \cdots) . \dagger
\end{aligned}
$$

Ich behaupte, dass die unendliche Koeffizientenfolge $c_{0}, c_{1}, \cdots, c_{\nu}, \cdots$ vollmonoton ist. Tatsächlich besteht zunächst $c_{v}$, laut (7), aus $n+1$, d.h. aus einer festen Anzahl von Faktoren. Irgend einer der $n$ ersten Faktoren hat die Form

$$
f_{\nu}^{(k)}=\frac{2 \nu+2 k}{2 \nu+2 k-1}=1+\frac{1}{2 \nu+2 k-1},
$$

wo $k$ eine feste positive ganze Zahl, und zwar eine der Zahlen 1, 2, $\cdots, n$, bezeichnet. Durchläuft nun $\nu$ die Zahlen $\nu=0,1,2,3, \cdots$, so erhalten wir eine vollmonotone Folge $f_{0}{ }^{(k)}, f_{1}{ }^{(k)}, f_{2}{ }^{(k)}, \cdots, f_{\nu}^{(k)}, \cdots$. Dies folgt aus dem Umstande, dass die Folge

$$
\frac{1}{2 \nu+2 k-1} \quad(\nu=0,1,2,3, \cdots)
$$

vollmonoton ist. Endlich liefert auch der $(n+1)$ te Faktor $f_{\nu}^{(n+1)}=(2 \nu+2 n+1)^{-1}$ eine vollmonotone Folge, wenn $\nu=0,1,2,3, \cdots$ gesetzt wird. Auf Grund von $\S I$ ist aber dann auch die Folge $f_{\nu}^{(1)} f_{\nu}^{(2)} \cdots f_{\nu}^{(n)} f_{\nu}^{(n+1)}, \nu=0,1,2,3, \cdots$, vollmonoton. (Diese Tatsache lässt natürlich auch noch verschiedene andere Beweise zu. Vgl. z.B. die Einleitung dieser Arbeit.)

Auf Grund des Satzes $§ I V$, Nr. 2, kann ich aus der blossen Tatsache, dass die Koeffizienten der Heinereihe für $(\pi / 4) P_{n}(\cos \theta)$ positiv sind, und monoton

*Z. B. lautet die Reihe für $n=0$

für $n=1$

$$
\frac{\pi}{4}=\sum_{\nu=0}^{\infty} \frac{\sin (2 \nu+1) \theta}{2 \nu+1}
$$

u. s. w.

$$
(\pi / 4) \cos \theta=\sum_{\nu=0}^{\infty} \frac{1}{2}\left(\frac{1}{2 \nu+1}+\frac{1}{2 \nu+3}\right) \sin 2(\nu+1) \theta,
$$

$\dagger$ Bezüglich einer anderen Darstellung von $c_{\nu}$ vgl. die Einleitung dieser Arbeit. 
abnehmen, was doch aus irgend einer von ihren Formen evident ist, den folgenden Satz aussprechen:

Teilt man das Intervall $(0, \pi)$ in $n$ gleiche Teile, so hat $P_{n}(\cos \theta)$ im Innern eines jeden Teiles eine Nullstelle. D.h. sind $t_{1}, t_{2}, \cdots, t_{n}$ die Nullstellen von $P_{n}(\cos \theta)$ im Intervalle $0<\theta<\pi$, so ist

$$
(m-1) \frac{\pi}{n}<t_{m}<m \frac{\pi}{n} \quad(m=1,2, \cdots, n) .
$$

Berücksichtigt man die Tatsache, dass die Koeffizientenfolge der Heineschen Sinusreihe dreifach monoton (ja sogar vollmonoton) ist, so liefert der Satz von §IV, Nr. 5:

Die Nullstellen $t_{m}$ von $P_{n}(\cos \theta)$, welche im Intervalle $0<\theta \leqq \pi / 2$ liegen, sind in die Teilintervalle

$$
\left(m-\frac{1}{2}\right) \frac{\pi}{n}<t_{m}<m \frac{\pi}{n+1} \quad\left(m=1,2,3, \cdots, n^{\prime}=\left[\frac{n+1}{2}\right]\right)
$$

eingeschlossen.

Ist $n$ ungerade, so muss statt der letzten dieser Ungleichungen

$$
\left(n^{\prime}-\frac{1}{2}\right) \frac{\pi}{n}=t_{n^{\prime}}=n^{\prime} \frac{\pi}{n+1} \quad\left(=\frac{\pi}{2}\right)
$$

stehen.

Dies ist die Markoff-Steiltjessche Einschränkung der Nullstellen des $n$ ten Legendreschen Polynoms.

2. Die konjugierte Kosinusreihe der Heineschen Reihe (6) stellt $\frac{1}{2} Q_{n}(x)$ $=\frac{1}{2} Q_{n}(\cos \theta)$, d.h. die Hälfte der $n$ ten Kugelfunktion zweiter Art auf dem Querschnitte $-1<x<+1$ dar. Es ist also

$$
\frac{1}{2} Q_{n}(\cos \theta)=\sum_{\nu=0}^{\infty} c_{\nu} \cos (n+2 \nu+1) \theta .
$$

Hier gibt die Zerlegung

$$
\begin{aligned}
\frac{1}{2} Q_{n}(\cos \theta) & =\left(\sum_{\nu=0}^{\infty} c_{\nu} \cos 2 \nu \theta\right) \cos (n+1) \theta \\
& -\left(\sum_{\nu=0}^{\infty} c_{\nu} \sin 2 \nu \theta\right) \sin (n+1) \theta,
\end{aligned}
$$

wegen $\sum_{\nu=0}^{\infty} c_{\nu} \cos 2 \nu \theta>0$ für $0<\theta<\pi$, dass

$$
\operatorname{sgn} Q_{n}\left(\cos \theta_{m}\right)=(-1)^{m}
$$

gilt, wenn $\theta_{m}=m \pi /(n+1), m=1,2, \cdots, n$, gesetzt wird. 
Daraus folgt: Teilt man das Intervall $(0, \pi)$ in $n+1$ gleiche Teile, so hat $Q_{n}(\cos \theta)$ im Innern eines jeden Teilintervalles mindestens eine Nullstelle, also insgesamt mindestens $n+1$ Nullstellen im Innern des Intervalls $(0, \pi)$.

3. Die Heinesche Reihe führt auch $z u$ anderen Eigenschaften der Legendreschen Polynome. So ergibt sich z.B. auch die sog. Stieltjessche Abschätzung mit einem Schlage aus der Form (5):

$$
f(\theta)=(\pi / 4) \alpha_{n}(2 n+1) P_{n}(\cos \theta)=\sum_{\nu=0}^{\infty} \alpha_{\nu} \beta_{\nu} \sin (n+2 \nu+1) \theta,
$$

wo

$$
\begin{array}{r}
\alpha_{0}=1, \quad \alpha_{\nu}=\frac{1 \cdot 3 \cdots(2 \nu-1)}{2 \cdot 4 \cdots 2 \nu}, \\
\beta_{0}=\beta_{0}^{(n)}=1, \quad \beta_{\nu}=\beta_{\nu}{ }^{(n)}=\frac{(2 n+2)(2 n+4) \cdots(2 n+2 \nu)}{(2 n+3)(2 n+5) \cdots(2 n+2 \nu+1)} \\
(\nu=1,2,3, \cdots) .
\end{array}
$$

Die Stieltjessche Abschätzung des Legendreschen Polynoms hat aus der Heineschen Reihe schon Hobson [14] abgeleitet. Die folgende Ableitung dürfte einfacher sein.

Da $f(\theta)$ die für $z=e^{i \theta}$ genommene imaginäre Komponente der Potenzreihe

$$
F(z)=\sum_{\nu=0}^{\infty} \alpha_{\nu} \beta_{\nu} z^{n+2 v+1}=z^{n+1} \sum_{\nu=0}^{\infty} \alpha_{\nu} \beta_{\nu} z^{2 v}
$$

darstellt, so ist also sicher

$$
|f(\theta)| \leqq\left|F\left(e^{i \theta}\right)\right|=\left|\sum_{\nu=0}^{\infty} \alpha_{\nu} \beta_{\nu} z^{\nu}\right|_{z=e^{2 i \theta}}, \quad 0<\theta<\pi .
$$

Da $\beta_{0}=1, \beta_{1}, \beta_{2}, \cdots$ eine einfach monotone Nullfolge ist, so liefert die Abelsche Umformung

$$
|f(\theta)| \leqq\left|\sum_{\nu=0}^{\infty}\left(\beta_{\nu}-\beta_{\nu+1}\right)\left(\alpha_{0}+\alpha_{1} z+\cdots+\alpha_{\nu} z^{\nu}\right)\right|_{z=e^{2 i \theta}} .
$$

Nun gilt aber für die Abschnitte der Binomialreihe für $(1-z)^{-1 / 2}$ [Fejér 6, Szegö 25]

$$
\begin{aligned}
\left|\alpha_{0}+\alpha_{1} z+\cdots+\alpha_{\nu} z^{\nu}\right| & \leqq 2^{1 / 2}|1-z|^{-1 / 2} \\
(\nu & =0,1,2,3, \cdots ;|z| \leqq 1, z \neq 1),
\end{aligned}
$$

also ist 


$$
|f(\theta)| \leqq 2^{1 / 2}|1-z|_{z=e^{2 i \theta}}^{-1 / 2}=(\sin \theta)^{-1 / 2},
$$

d.h.

$$
\begin{aligned}
\frac{\pi}{4} \frac{1 \cdot 3 \cdots(2 n-1)}{2 \cdot 4 \cdots 2 n}(2 n+1) \mid & P_{n}(\cos \theta) \mid \leqq(\sin \theta)^{-1 / 2} \\
& (0<\theta<\pi, n=0,1,2,3, \cdots) .
\end{aligned}
$$

Das ist die Stieltjessche Abschätzung für $P_{n}(\cos \theta)$. Wir sehen, dass sie auch für die konjugierte Kosinusreihe der Heineschen Sinusreihe, d.h. für $\frac{1}{2} Q_{n}(\cos \theta)$ statt $(\pi / 4) P_{n}(\cos \theta)$ gültig ist.

4. Da die $\alpha_{\nu}, \nu=0,1,2, \cdots$, von $n$ unabhängig sind und eine monotone Nullfolge bilden, da ferner auch die Folge $1=\beta_{0}^{(n)}, \beta_{1}^{(n)}, \cdots, \beta_{\nu}^{(n)}, \cdots$ eine monotone (Nullfolge) mit $\lim _{n \rightarrow \infty} \beta_{\nu}(n)=1$ bei beliebigem, aber festem, $\nu$ ist, da schliesslich die Reihe $\sum_{\nu=0}^{\infty} \alpha_{\nu} z^{2 \nu}, z=e^{i \theta}$, auf jedem Bogen $\epsilon \leqq \theta \leqq \pi-\epsilon, \epsilon>0$, des oberen Halbeinheitskreises, nach den Sätzen von Schlömilch und Abel, gleichmässig gegen ihre Summe $\left(1-e^{2 i \theta}\right)^{-1 / 2}$ konvergiert, so ist auch

$$
\begin{aligned}
& \lim _{n \rightarrow \infty}\left(\sum_{\nu=0}^{\infty} \alpha_{\nu} \beta_{\nu}^{(n)} e^{2 i \theta \nu}\right)=\left(1-e^{2 i \theta}\right)^{-1 / 2} \\
& \quad=(2 \sin \theta)^{-1 / 2} \cos (\pi / 4-\theta / 2)+i \cdot(2 \sin \theta)^{-1 / 2} \sin (\pi / 4-\theta / 2),
\end{aligned}
$$

und zwar gleichmässig auf jedem Bogen $\epsilon \leqq \theta \leqq \pi-\epsilon, \epsilon>0$. Somit liefert (18)

$$
\begin{aligned}
(\pi / 4) \alpha_{n}(2 n+1) P_{n}(\cos \theta) & =(2 \sin \theta)^{-1 / 2}\left\{\cos \left[\left(n+\frac{1}{2}\right) \theta-\pi / 4\right]+\delta_{n}(\theta)\right\}, \\
\frac{1}{2} \alpha_{n}(2 n+1) Q_{n}(\cos \theta) & =(2 \sin \theta)^{-1 / 2}\left\{\cos \left[\left(n+\frac{1}{2}\right) \theta+\pi / 4\right]+\eta_{n}(\theta)\right\},
\end{aligned}
$$

wo

$$
\lim _{n \rightarrow \infty} \delta_{n}(\theta)=\lim _{n \rightarrow \infty} \eta_{n}(\theta)=0,
$$

und zwar gleichmässig für jedes Teilintervall $\epsilon \leqq \theta \leqq \pi-\epsilon, \epsilon>0$. (25) ist die Laplacesche asymptotische Formel für $P_{n}(\cos \theta)$, (26) die Heinesche für $Q_{n}(\cos \theta)$. [Siehe Heine 13, Bd. 1, S. 175.]

VII. Asymptotische Eigenschaften des Restes einer beliebigen Fourierschen Sinusreihe. Präzisierung des Resultates

IM FALLE EINER EINFACH ODER MEHRFACH MONOTONEN KOEFFIZIENTENFOLGE

1. Es sei $f(\theta)$ eine beliebige, im Intervalle $0 \leqq \theta \leqq \pi$ definierte stetige Funktion, deren Ableitung daselbst ebenfalls stetig ist. Es sei

$$
f(\theta)=b_{1} \sin \theta+b_{2} \sin 2 \theta+\cdots+b_{n} \sin n \theta+\cdots
$$

die Fouriersche Sinusreihe dieser Funktion, d.h. 


$$
b_{n}=\frac{2}{\pi} \int_{0}^{\pi} f(t) \sin n t d t \quad(n=1,2,3, \cdots) .
$$

Mit Hilfe der Methode die ich in meiner Arbeit über den Rest der Fourierschen Reihe angegeben habe [Fejér 2, 3, 4] findet man leicht für den Rest

$$
f(\theta)-s_{n}(\theta)=\sum_{\nu=n+1}^{\infty} b_{\nu} \sin \nu \theta, \quad 0<\theta<\pi,
$$

die folgende asymptotische Formel:

$$
\begin{aligned}
f(\theta)-s_{n}(\theta)= & \frac{1}{\left(n+\frac{1}{2}\right) \pi}\left\{f(+0) \frac{\cos \left(n+\frac{1}{2}\right) \theta}{\sin \frac{1}{2} \theta}\right. \\
& \left.+(-1)^{n} f(\pi-0) \frac{\sin \left(n+\frac{1}{2}\right) \theta}{\cos \frac{1}{2} \theta}+\epsilon_{n}(\theta)\right\},
\end{aligned}
$$

wo $0<\theta<\pi$ und $\epsilon_{n}(\theta)$ im Intervalle $\delta \leqq \theta \leqq \pi-\delta$ für beliebig kleines, aber festes positives $\delta$ gleichmässig gegen 0 konvergiert. ${ }^{*}$ Ich setze jetzt voraus, dass mindestens einer der beiden Werte $f(+0), f(\pi-0)$, etwa $f(+0)$, von 0 verschieden sei.

Wir betrachten nun die Wurzeln der Gleichung $\sin \left(n+\frac{1}{2}\right) \theta=0$, d.h.

$$
\theta_{m}=2 m \frac{\pi}{2 n+1} \quad(m=1,2,3, \cdots),
$$

von diesen jedoch nur diejenigen, die in das Intervall $\delta \leqq \theta \leqq \pi-\delta$ fallen, wo $\delta$ eine feste positive Grösse bezeichnet. Da aus (4)

$$
\left(f(\theta)-s_{n}(\theta)\right)_{\theta=\theta_{m}}=\frac{1}{\left(n+\frac{1}{2}\right) \pi}\left\{\frac{f(+0)}{\sin \left(\theta_{m} / 2\right)} \cos m \pi+\epsilon_{n}\left(\theta_{m}\right)\right\}
$$

folgt, so hat man, wegen der gleichmässigen Konvergenz von $\epsilon_{n}(\theta)$ gegen 0 , wenn nur $n$ geriugend gross ist:

$$
\operatorname{sgn}\left(f(\theta)-s_{n}(\theta)\right)_{\theta=\theta_{m}}=(-1)^{m} \operatorname{sgn} f(+0) .
$$

Wir haben also für den Rest

* Für

lautet die Formel

$$
f(\theta)=\frac{\pi-\theta}{2}=\frac{\sin \theta}{1}+\frac{\sin 2 \theta}{2}+\cdots+\frac{\sin n \theta}{n}+\cdots
$$

$$
\frac{\sin (n+1) \theta}{n+1}+\frac{\sin (n+2) \theta}{n+2}+\cdots=\frac{1}{2 n+1}\left\{\frac{\cos \left(n+\frac{1}{2}\right) \theta}{\sin \frac{1}{2} \theta}+\eta_{n}(\theta)\right\},
$$

wo $\lim _{n \rightarrow \infty} \eta_{n}(\theta)=0$, und zwar gleichmässig für $\delta \leqq \theta \leqq \pi-\delta, \delta>0$. 


$$
R_{n+1}(\theta)=f(\theta)-s_{n}(\theta)=\sum_{\nu=n+1}^{\infty} b_{\nu} \sin \nu \theta
$$

das folgende Resultat erhalten:

Bewegt sich $\theta$ nur im Intervalle $\delta \leqq \theta \leqq \pi-\delta$, wo $\delta$ eine beliebig kleine, aber feste positive Zahl bezeichnet, ist ferner der Index $n$ entsprechend gross, so besitzt der Rest (3) der Fourierschen Sinusreihe der den obigen Bedingungen genügenden beliebigen Funktion $f(\theta)$ mindestens eine Wurzel zwischen je $z$ wei aufeinanderfolgenden Wurzeln

$$
\begin{aligned}
\theta_{m-1} & =2(m-1) \frac{\pi}{2 n+1}, \\
\theta_{m} & =2 m \frac{\pi}{2 n+1}
\end{aligned}
$$

der Gleichung $\sin \left(n+\frac{1}{2}\right) \theta=0$, falls diese beiden Wurzeln in das Intervall $\delta \leqq \theta \leqq \pi-\delta$ fallen.

Wie kann dieser Satz vom Sturmschen Charakter präzisiert werden im Spezialfalle, wo die Koeffizientenfolge der Sinusreihe

$$
b_{1} \sin \theta+b_{2} \sin 2 \theta+\cdots+b_{n} \sin n \theta+\cdots
$$

eine einfach monotone Nullfolge ist? Insofern ist jetzt unsere Annahme wieder etwas allgemeiner, als es gar nicht nötig ist, dass die Reihe (8) eine Fouriersche sei. Die Zerlegung (4) in $\S \mathrm{V}, \mathrm{Nr}$. 1, gibt uns ohne weiteres den folgenden Satz:

Ist die Koeffizientenfolge der unendlichen Sinusreihe (8) eine beliebige einfach monotone Nullfolge, so hat der Rest

$$
b_{n+1} \sin (n+1) \theta+b_{n+2} \sin (n+2) \theta+\cdots
$$

mindestens $n$ voneinander verschiedene Nullstellen (Zeichenwechselstellen) $t_{1}, t_{2}, \cdots, t_{n}$ im Innern des Intervalles $(0, \pi)$, und zwar ist

$$
2(m-1) \frac{\pi}{2 n+1}<t_{m}<2 m \frac{\pi}{2 n+1} \quad(m=1,2, \cdots, n) .
$$

Dies gilt für jeden Wert von $n$.

Mit anderen Worten: Was im Falle einer "beliebigen" Reihe (a) nur in einem Teilintervalle von $(0, \pi)$, und (b) nur für genügend grosse Werte von $n$ gültig ist, ist im Falle monoton abnehmender Koeffizienten im ganzen Intervalle $(0, \pi)$ und für jedes $n$ gültig. 
VIII. Abschätzung des Restes und der Partialsumme einer PotenzReihe Mit Hilfe der dargestellten Funktion, WENN DIE KOEFFIZIENTENFOLGE EINFACH ODER MEHRFACH MONOTON IST

1. Essei

$$
c_{0}, c_{1}, c_{2}, \cdots, c_{n}, \cdots
$$

eine beliebige dreifach monotone Nullfolge. Dann ist die Potenzreihe

$$
f(z)=\sum_{\nu=0}^{\infty} c_{\nu} z^{\nu}
$$

für $|z| \leqq 1, z \neq 1$, konvergent und es gilt für die Restsumme $R_{n}(z)=\sum_{\nu=n+1}^{\infty} c_{\nu} z^{\nu}$

$$
\left|R_{n}(z)\right| \leqq|f(z)|, \text { fïr }|z| \leqq 1, z \neq 1, n=0,1,2, \cdots .
$$

Für den absoluten Betrag der Partialsummen $s_{n}(z)$ ist also

$$
\left|s_{n}(z)\right|=\left|c_{0}+c_{1} z+\cdots+c_{n} z^{n}\right| \leqq 2|f(z)|
$$

gültig für $|z| \leqq 1, z \neq 1, n=0,1,2, \cdots$.

Beweis. Da

$$
R_{n}(z)=\sum_{\nu=0}^{\infty} c_{n+\nu} z^{n+\nu}
$$

so ist für $z=r e^{i \theta}, 0 \leqq r<1$,

$$
R_{n}\left(r e^{i \theta}\right)=\sum_{\nu=0}^{\infty} c_{n+\nu} r^{n+\nu} e^{i(n+\nu) \theta}=e^{i n \theta} \sum_{\nu=0}^{\infty} c_{n+\nu} r^{n+\nu} e^{i \nu \theta} .
$$

Da $c_{n+\nu} r^{n+\nu}$ bei festem $r$ hinreichend stark gegen 0 konvergiert, wenn $\nu \rightarrow \infty$, so ist die Abelsche Umformung zweiter Ordnung gestattet:

$$
R_{n}\left(r e^{i \theta}\right)=e^{i n \theta} \sum_{\nu=0}^{\infty} \Delta^{2}\left(c_{n+\nu} r^{n+\nu}\right)\left(s_{\nu}^{(1)}(\theta)+i \sigma_{\nu}^{(1)}(\theta)\right),
$$

wo $s_{\nu}^{(1)}(\theta)$ die Partialsummen erster Ordnung der Reihe

$$
1+\cos \theta+\cos 2 \theta+\cdots+\cos n \theta+\cdots,
$$

$\sigma_{\nu}^{(1)}(\theta)$ die Partialsummen erster Ordnung der Reihe

$$
0+\sin \theta+\sin 2 \theta+\cdots+\sin n \theta+\cdots
$$

bezeichnen. [Es ist also

$$
\begin{aligned}
& s_{\nu}^{(1)}(\theta)=(\nu+1)+\nu \cos \theta+\cdots+1 \cdot \cos \nu \theta ; \\
& \left.\sigma_{\nu}^{(1)}(\theta)=\nu \sin \theta+(\nu-1) \sin 2 \theta+\cdots+1 \cdot \sin \nu \theta .\right]
\end{aligned}
$$


Da ferner die Folge $\left\{c_{\nu}\right\}$ als dreifach monoton vorausgesetzt wurde, so ist sie jedenfalls auch zweifach monoton, und die Folge $\left\{c_{\nu} r^{r}\right\}$ ist, wegen $r<1$, ebenfalls zweifach monoton. Das gleiche trifft für die Restfolge der letzteren Folge, d.h. für

$$
c_{n} r^{n}, c_{n+1} r^{n+1}, \cdots
$$

zu. Weiter sind $s_{\nu}^{(1)}(\theta)$ und $\sigma_{\nu}^{(1)}(\theta)$ nichtnegativ für $0<\theta<\pi$ und $\nu=0,1$, $2,3, \ldots$. Somit habe ich also gezeigt, dass in der unendlichen Summe auf der rechten Seite der Gleichung (7) [die den Wert $e^{-i n \theta} R_{n}\left(r e^{i \theta}\right)$ hat] jedes Glied eine komplexe Zahl mit nichtnegativer reeller und imaginärer Komponente ist, $0<\theta<\pi$.

Die Folge $\left\{c_{\mu}\right\}$, also auch $\left\{c_{\mu} r^{\mu}\right\}$, ist aber sogar dreifach monoton, so dass die zweiten Differenzen

$$
\Delta^{2}\left(c_{0} r^{0}\right), \Delta^{2}\left(c_{1} r^{1}\right), \Delta^{2}\left(c_{2} r^{2}\right), \cdots, \Delta^{2}\left(c_{\mu} r^{\mu}\right), \cdots
$$

nicht nur nichtnegativ, sondern auch monoton abnehmend sind. Ersetze ich also in der Summe für $e^{-i n \theta} R_{n}\left(r e^{i \theta}\right)$ die Differenz $\Delta^{2}\left(c_{n+\nu} r^{n+\nu}\right)$, für jedes $\nu$, durch die Differenz $\Delta^{2}\left(c_{\nu} r^{\nu}\right)$, so wird der absolute Betrag der unendlichen Summe nicht verkleinert, d.h.

$$
\left|R_{n}\left(r e^{i \theta}\right)\right| \leqq\left|\sum_{\nu=0}^{\infty} \Delta^{2}\left(c_{\nu} r^{\nu}\right)\left(s_{\nu}^{(1)}(\theta)+i \sigma_{\nu}^{(1)}(\theta)\right)\right| .
$$

Was aber jetzt rechts innerhalb des Zeichens des absoluten Betrages steht, ist $f\left(r e^{i \theta}\right)$ selbst [in Abelscher Umformung zweiter Ordnung ihrer Summenform (2) ]. Also haben wir

$$
\left|R_{n}\left(r e^{i \theta}\right)\right| \leqq\left|f\left(r e^{i \theta}\right)\right| .
$$

Dies ist zunächst für $0<\theta<\pi$ abgeleitet worden; wegen der Realität der $c_{\text {v }}$ ist es jedoch für jedes reelle $\theta$ gültig. Hierbei wurde stets $r<1$ vorausgesetzt. Da nun die Reihen $\sum_{\nu=0}^{\infty} c_{\nu} z^{\nu}, \sum_{\nu=n+1}^{\infty} c_{\nu} z^{\nu}$ auch für $z=e^{i \theta}$ überall konvergent sind (mit eventueller Ausnahme von $z=1$, d.h. $\theta=0$ ), so habe ich also schliesslich (3) bewiesen, woraus wegen $s_{n}(z)=f(z)-R_{n+1}(z)$, wie schon bemerkt, (4) folgt. [Siehe: M. Riesz 23, S. Chapman 1, L. Fejér 6, G. Szegö 25. Man vgl. das allgemeine Kriterium von Szegö mit dem meinigen.]

IX. Über die Schlichtheit von PotenzReihen mit monotoner KOEFFIZIENTENFOLGE VERSCHIEDENER ORDNUNG

\section{Ist in der Potenzreihe}

$$
f(z)=c_{1} z+c_{2} z^{2}+\cdots+c_{n} z^{n}+\cdots
$$

die Koeffizientenfolge $\left\{c_{n}\right\}$ vierfach monoton, so bildet sie das Innere des Einheitskreises $|z|<1$ schlicht auf die Funktionsebene ab. 
Beweis. Es ist für $r<1$

$$
f\left(r e^{i \theta}\right)^{\cdot}=u(r, \theta)+i v(r, \theta)=\left(\sum_{\nu=0}^{\infty} c_{\nu} r^{\nu} \cos \nu \theta\right)+i\left(\sum_{\nu=0}^{\infty} c_{\nu} r^{\nu} \sin \nu \theta\right) .
$$

$\mathrm{Da}\left\{c_{\nu} \boldsymbol{r}^{\nu}\right\}, \nu=1,2,3, \cdots$, gewiss zweifach monoton ist, so gilt, nach dem Satze in §III, Nr. 3, v(r, $\theta)>0$ für $0<\theta<\pi$. Da $\left\{c_{\nu} r^{\nu}\right\}, \nu=1,2,3, \cdots$, auch noch vierfach monoton ist, so ist $u(r, \theta)$ monoton fallend für $0<\theta<\pi$ (§III, Nr. 2). Wenn also der Punkt $r e^{i \theta}$ bei festem $r<1$ den oberen Halbkreis $r e^{i \theta}$, $0<\theta<\pi$, beschreibt, von $\theta=0$ angefangen bis $\theta=\pi$, so beschreibt der Bildpunkt $u(r, \theta)+i v(r, \theta)$ eine, in der oberen Halbebene gelegene Kurve der $w$-Ebene, die keinen Doppelpunkt haben kann. Mit Rücksicht auf den Umstand, dass das Kreisbild $f\left(r e^{i \theta}\right), 0 \leqq \theta<2 \pi$, symmetrisch in Bezug auf die $u$-Achse ist, habe ich also bewiesen, dass jedem Kreise $|z|=r, r<1$, eine Jordankurve in der Funktionsebene entspricht, woraus unser Satz folgt.

Weiter ist der folgende Satz gültig.

\section{Ist in der Potenzreihe}

$$
f(z)=c_{1} z+c_{2} z^{3}+c_{3} z^{5}+\cdots+c_{\nu} z^{2 \nu-1}+\cdots
$$

die Koeffizientenfolge $\left\{c_{\nu}\right\}$ dreifach monoton, so ist sie für $|z|<1$ konvergent und schlicht.

2. Die Funktionen $f(z)$ wie (1) und (3) bilden eine Unterklasse derjenigen im Einheitskreise schlichten Funktionen, die ich in meinen Noten [L. Fejér $9,10]$ betrachtet habe, und die allgemein dadurch charakterisiert werden können, dass für sie $z f^{\prime}(z)$ im Innern der oberen Hälfte des Einheitskreises $\left(z=r e^{i \theta}, 0<r<1,0<\theta<\pi\right)$ eine imaginäre Komponente beständigen Vorzeichens hat.* Auf Grund der Resultate meiner Noten kann ich also noch hinzufügen, dass bei der Reihe (1) die arithmetischen Mittel dritter Ordnung der Partialsummen, bei der Reihe (3) die arithmetischen Mittel zweiter Ordnung der Partialsummen für $|z|<1$ und für $n=0,1,2,3, \cdots$, schlicht sind.

\section{LITERATURVERZEICHNIS}

1. Chapman, S. On non-integral orders of summability of series and integrals. Proceedings of the London Mathematical Society, (2), vol. 9 (1910), pp. 369-409.

2. Fejér, L. Sur la série de Fourier. Comptes Rendus, Paris, vol. 142 (1906), pp. 501-503.

* Diese Klasse ist tatsächlich umfangreicher, wie das Beispiel

$$
f(z)=8 z+z^{2}+2 z^{3}=u(r, \theta)+i v(r, \theta)
$$

zeigt. Hier ist $v(r, \theta)>0, d u(r, \theta) / d \theta<0$ für $0<r \leqq 1,0<\theta<\pi$. Folglich ist $f(z)$ schlicht für $|z| \leqq 1$ und gehött zu unserer allgemeineren Klasse, nicht aber zur jetzt betrachteten speziellen, da doch $8,1,2$, $0,0,0, \cdots$ nicht einmal monoton ist. 
3. - A Fourier-féle sorról. Matematikai és Természettudományi Értesitö, Budapest, vol. 24 (1906), pp. 292-297, 369-390.

4. — Über die Fouriersche Reihe. Mathematische Annalen, vol. 64 (1907), pp. 273-288.

5. — Über die Positivität von Summen, die nach trigonometrischen oder Legendreschen Funktionen fortschreiten. I. Acta Litterarum ac Scientiarum, Szeged, vol. 2 (1925), pp. 75-86.

6. - Abschätzungen für die Legendreschen und verwandte Polynome. Mathematische Zeitschrift, vol. 24 (1925), pp. 285-298.

7. - Einige Sätze, die sich auf das Vorzeichen einer ganzen rationalen Funktion beziehen; nebst A nwendungen etc. Monatshefte für Mathematik und Physik, vol. 35 (1928), pp. 305-344.

8. - Über ein trigonometrisches Analogon eines Kakeyaschen Satzes. Jahresbericht der Deutschen Mathematiker-Vereinigung, vol. 38 (1929).

9. - Gestaltliches über die Partialsummen und ihrer Mittelwerte bei der Fourierreihe und der Polenzreihe. Zeitschrift für angewandte Mathematik und Mechanik, vol. 13 (1933).

10. - Neue Eigenschaften der Mittelwerte bei den Fourierreihen. Journal of the London Mathematical Society, vol. 8 (1933), pp. 53-62.

11. Hausdorff, F. Summationsmethoden und Momentfolgen. I. II. Mathematische Zeitschrift, vol. 9 (1921), pp. 74-109, 280-299.

12. - Momentprobleme für ein endliches Intervall. Mathematische Zeitschrift, vol. 16 (1923), pp. 220-248.

13. Heine, E. Theorie der Kugelfunktionen und der verwandten Funktionen. 2te Auflage, Berlin, 1878.

14. Hobson, E. W. On the representation of a function by a series of Legendre's functions. Proceedings of the London Mathematical Society, (2), vol. 7 (1909), pp. 24-39.

15. Hurwitz, A. Über die Fourierschen Konstanten integrierbarer Funktionen. Mathematische Annalen, vol. 57 (1903), pp. 425-446.

16. Jacobsthal, E. Miltelwertbildung und Reihentransformation. Mathematische Zeitschrift, vol. 6 (1920), pp. 110-117.

17. Kaluza, Th. Struktur und Eigenschaften mehrfach monotoner Folgen. Mathematische Zeitschrift, vol. 26 (1927), pp. 345-364.

18. Knopp, K. Mittelwertbildung und Reihentransformation. Mathematische Zeitschrift, vol. 6 (1920), pp. 118-123.

19. - Über das Eulersche Summierungsverfahren. Mathematische Zeitschrift, vol. 15 (1922), pp. 226-253; vol. 18 (1923), pp. 125-156.

20. - Neuere Untersuchungen in der Theoric der divergenten Reihen. Jahresbericht der Deutschen Mathematiker-Vereinigung, vol. 32.

21. —_ Mchrfach monotone Zahlenfolgen. Mathematische Zeitschrift, vol. 22 (1925), pp. 7585.

22. Pólya, G. Über dic Nullstellen gewisser ganzer Funktionøn. Mathematische Zeitschrift, vol. 2 (1918), pp. 352-383.

23. Riesz, M. Sur la sommation des séries de Fourier. Acta Litterarum ac Scientiarum, Szeged, vol. 1 (1923), pp. 104-113.

24. Stieltjes, T. J. Sur les polynomes de Legendre. CEuvres Complètes, vol. 2, pp. 234-252, Groningen, 1918.

25. Szegö, G. Bemerkungen zu einer Arbeit von Herrn Fcjér über die Legendreschen Polynome. Mathematische Zeitschrift, vol. 25 (1926), pp. 172-187.

26. - Inequalities for the seros of Legendre polynomials and related functions. These Transactions, vol. 39 (1936), pp. 1-17.

UNIVERSITy OF BUdAPEST,

Budapest, Hung.ARY 